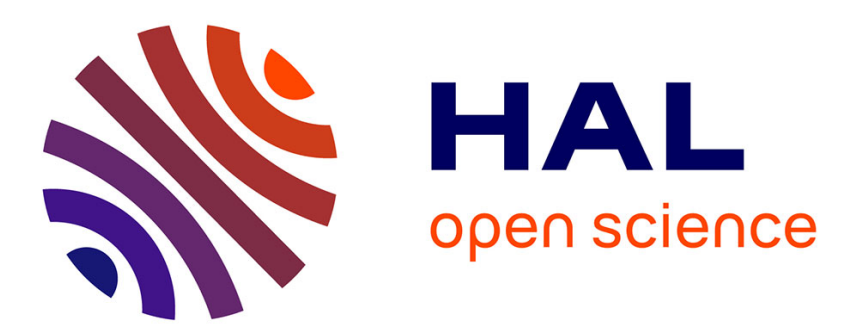

\title{
Atomic-based phase-field method for the modeling of radiation induced segregation in $\mathrm{Fe}-\mathrm{Cr}$
}

\author{
J.B. Piochaud, M. Nastar, F. Soisson, L. Thuinet, A. Legris
}

\section{To cite this version:}

J.B. Piochaud, M. Nastar, F. Soisson, L. Thuinet, A. Legris. Atomic-based phase-field method for the modeling of radiation induced segregation in $\mathrm{Fe}-\mathrm{Cr}$. Computational Materials Science, 2016, 122, pp.249-262. 10.1016/j.commatsci.2016.05.021 . cea-02389670

\section{HAL Id: cea-02389670 https://hal-cea.archives-ouvertes.fr/cea-02389670}

Submitted on 2 Dec 2019

HAL is a multi-disciplinary open access archive for the deposit and dissemination of scientific research documents, whether they are published or not. The documents may come from teaching and research institutions in France or abroad, or from public or private research centers.
L'archive ouverte pluridisciplinaire HAL, est destinée au dépôt et à la diffusion de documents scientifiques de niveau recherche, publiés ou non, émanant des établissements d'enseignement et de recherche français ou étrangers, des laboratoires publics ou privés. 


\title{
Atomic-based phase-field method for the modeling of radiation induced segregation in $\mathrm{Fe}-\mathrm{Cr}$
}

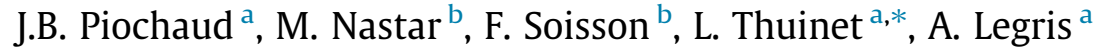 \\ a Univ. Lille, CNRS, INRA, ENSCL, UMR 8207, UMET, Unité Matériaux et Transformations, F 59000 Lille, France \\ b DEN-Service de Recherches de Métallurgie Physique, CEA, Université Paris-Saclay, F-91191 Gif-sur-Yvette, France
}

\section{A R T I C L E I N F O}

\section{Article history:}

Received 2 March 2016

Received in revised form 17 May 2016

Accepted 18 May 2016

Available online 6 June 2016

\section{Keywords:}

Radiation induced segregation

Iron-chromium alloys

Phase-field model

Monte Carlo simulations

\begin{abstract}
A B S T R A C T
We present a quantitative phase-field modeling of radiation-induced segregation in $\mathrm{Fe}-\mathrm{Cr}$ alloys. The evolution of chemical and point defect concentration fields are described by an Onsager formalism combined to a Cahn-Hilliard like diffusion equation for the introduction of non-uniform driving forces. Both the Onsager transport coefficients and the driving force parameters are extracted from atomic Monte Carlo simulations with point defect diffusion models fitted on DFT calculations, in a composition range between 0 and 20 at.\% $\mathrm{Cr}$ and in a temperature range between 600 and $1000 \mathrm{~K}$. Phase-field simulations are able to quantitatively reproduce the evolution of segregation profiles obtained from direct atomistic kinetic Monte Carlo simulations, while being typically two orders of magnitude faster. It is shown that a precise parameterization of the concentration-dependent Onsager transport coefficients, thermodynamic factors, and equilibrium point defect concentrations is crucial for the phase-field method to be quantitative.
\end{abstract}

(c) 2016 Elsevier B.V. All rights reserved.

\section{Introduction}

Materials under irradiation are maintained in non-equilibrium conditions that can accelerate or even induce microstructure changes. In particular, radiation induced segregation (RIS) occurs when net fluxes of point defects (PDs) are driven by irradiation. The latter are due to the creation and subsequent diffusion of PDs such as vacancies and interstitials toward PD sinks such as grain boundaries or dislocation loops (see Refs. [1-3] for recent reviews). This mechanism leads to a local chemical redistribution in the vicinity of sinks, which typically happens at temperatures between 0.3 and 0.6 times the melting point. RIS is very common in alloys and has an impact on the evolution of their mechanical properties and corrosion resistance under irradiation. In particular, it has important technological implications in materials used in the nuclear industry such as austenitic and ferritic steels.

RIS results from the coupling between the fluxes of excess vacancies and interstitials generated under irradiation, and the fluxes of chemical elements (here $\mathrm{Fe}$ and $\mathrm{Cr}$ ). Thermodynamics of irreversible processes shows that the modeling of these couplings requires a reliable description of (i) the chemical potentials and (ii) the $L$-coefficients (i.e. the full Onsager matrices for interstitial

\footnotetext{
* Corresponding author.

E-mail address: Ludovic.Thuinet@univ-lille1.fr (L. Thuinet).
}

and vacancy diffusion, including the off-diagonal terms), and of their variation with PD concentrations and local alloy concentrations [2,4]. However, experimental measurements of the L-coefficients of an alloy are limited, especially in concentrated and multicomponent alloys. To address this issue, most of the RIS models for concentrated alloys (including the well known Perks model [5]) used empirical expressions of the $L$-coefficients in terms of tracer diffusion coefficients. Some of the kinetic and thermodynamic couplings have hence been ignored. In particular, due to the lack of diffusion data for the interstitials, most of the first RIS models emphasized the vacancy contribution. Moreover, the thermodynamic quantities were mainly assumed to be the ones of an ideal stress-free solid solution whereas they are expected to significantly depend on the local composition and stress field [6,7].

Over the years, the description of kinetic flux couplings has been improved. The $L$-coefficients are now currently derived from mean-field diffusion theories taking as input the $a b$ initio calculations of the PD migration energies with respect to the local alloy composition. Recent progress has been mainly achieved in the limit case of dilute alloys [8-11]. However beyond the dilute limit, a quantitative mean-field model of RIS including flux couplings and consistently defined thermodynamic parameters is still missing.

Atomistic Kinetic Monte Carlo (AKMC) modeling has also been used to simulate RIS, since correlation effects between successive PD jumps, flux couplings as well as thermal fluctuations, are 
naturally included into it $[12,13]$. Thus, they provide an accurate description of diffusion properties, provided that a good model of PD jump frequencies is used. The method has been used to study RIS in $\mathrm{Fe}-\mathrm{Cr}$ alloys, using pair interactions fitted on $a b$ initio calculations to compute the jump frequencies [13]. However, AKMC simulations are time consuming compared to mean field models, especially when correlation and trapping effects are significant, which reduces their scope of applications. They can only simulate relatively small systems of the order of a few tens of nanometers and cannot easily take into account elasticity. Moreover, in present AKMC simulations of RIS, the production of point defects at sinks is neglected, leading to zero equilibrium PD concentrations. Up to now, the role of source of a PD sink has been simulated in dilute alloys only [14].

In that context, RIS modeling inevitably requires a multiscale approach in order to bridge the gap between physics at the atomic scale and the mesoscopic scale at which systems self-organize. Previous Phase Field (PF) models have been proposed to study the kinetics of segregation and precipitation in alloys under irradiation by Badillo et al. [15] and by Dubey and El-Azab [16], the first one focuses on point defect and solute clustering with a partial description of flux coupling, the second one gives a phenomenological description of flux couplings and RIS in model alloys. PF models of other irradiation effects have been reviewed in [17]. The aim of this paper is to present a quantitative phase-field model of RIS in $\mathrm{Fe}-\mathrm{Cr}$ alloys, starting from an atomic description of the jump frequencies. At the atomic scale, AKMC models fitted on ab initio calculations provide reliable data for the variation of atomic jump frequencies with local concentration. The $L$-coefficients and the chemical potentials of alloying elements and point defects are determined by AKMC simulations at various temperatures and compositions. The PF is then used to model the evolution under irradiation of PD and solute concentration profiles, in the vicinity of a grain boundary.

An extension of the PF method dedicated to the modeling of RIS in alloys is introduced. The equations are based on the Onsager formalism combined to the Cahn-Hilliard $(\mathrm{CH})$ model [18] of the thermodynamic alloy driving force and the self-consistent mean field (SCMF) theory for the definition of the non-uniform kinetic contribution [19]. This new PF model based on generalized diffusion equations takes into account the flux couplings between the atoms and PDs, the alloy and PD thermodynamic driving forces, the creation, the recombination and the elimination of PDs at sinks. All the thermodynamic and kinetic properties that are required to capture the complexity of the RIS mechanism have been calculated using AKMC simulations based on the model and parameters of Senninger et al. [13]. These simulations have been shown to be in agreement with diffusion experiments and consistent with RIS observations performed in industrial $\mathrm{Fe}-\mathrm{Cr}$ alloys. The thermodynamic and kinetic properties are calculated in a fraction range of $0-20$ at.\% $\mathrm{Cr}$ and in a temperature range of $600-1000 \mathrm{~K}$, which are then used as input parameters in the PF model. We restrict ourselves to the relatively simple situation where only segregation occurs, without precipitation of the $\alpha^{\prime}$ (Cr-rich) phase, because the classical kinetic properties are not well defined quantities within a two-phase equilibrium state, making the analysis of RIS more difficult. Moreover, PF approaches are well suited to the study of growth, coarsening and spinodal decomposition regimes, but not to the nucleation of precipitates in metastable solid solutions unless size-dependent fluctuations are introduced [20-22].

The objective is first to demonstrate the validity of the PF model by performing a systematic comparison with direct AKMC simulations of RIS, second to investigate the role of each thermodynamic parameter separately, a study that cannot be easily done in AKMC simulations. Moreover, the PF method will be used to simulate large systems and different sink densities.
The article is organized as follows. In Section 2, we present the computational details of the PF model. In particular, we detail the kinetic equations used in the PF model to simulate RIS and we propose a PF algorithm able to deal with species having very different diffusion coefficients. We emphasize both the kinetic and thermodynamic properties required to solve the PF equations. In Section 3 , a validation of the PF model is done by performing direct comparison with AKMC calculations. In Section 4, the influence of physical parameters used as input data of the PF simulations is discussed.

\section{Methods}

\subsection{Phase-field modeling}

\subsubsection{Kinetic equations}

The evolution of atomic and PD fractions as a function of time is modeled using $\mathrm{CH}$-like equations, which have been modified to consider (1) the PD creation induced by irradiation and characterized by the PD generation rate $K_{0}$, which is assumed to be constant and uniform, (2) the PD absorption at sinks represented by absorption rates $K_{d}^{a b s}$, (3) the recombination rate between the vacancies and the interstitials $K_{R}$, and (4) the kinetic contribution to the non-uniform alloy driving force. Five distinct order parameters are introduced in the kinetic equations:

(1) The fields of vacancy and interstitial site fractions, respectively $X_{\mathrm{V}}(r)$ and $X_{\mathrm{I}}(r)$, hereafter indicated with a subscript $d=\mathrm{I}, \mathrm{V}$, and the fields of $\mathrm{Fe}$ and $\mathrm{Cr}$ site fractions, respectively $X_{\mathrm{Fe}}(r)$ and $X_{\mathrm{Cr}}(r)$, hereafter indicated with the subscripts $\alpha=\mathrm{Fe}, \mathrm{Cr}$ or $\beta=\mathrm{Fe}, \mathrm{Cr}$.

(2) The field of PD sinks is represented by the order parameter $\lambda(r)$, equal to 0 in the matrix and 1 in the capture zone of the sink. This parameter allows a precise control of the sink geometry, which is essential to correctly calculate the sink strength.

The variation with time of chemical and point defect site fractions is given by

$$
\begin{aligned}
& \frac{\partial X_{d}(r, t)}{\partial t}=-V_{a t} \nabla \cdot \mathcal{J}_{d}(r, t)+K_{0}-K_{d}^{a b s}(r, t)-K_{R}(r, t) \\
& \frac{\partial X_{\alpha}(r, t)}{\partial t}=-V_{a t} \nabla \cdot \mathcal{J}_{\alpha}(r, t),
\end{aligned}
$$

where $V_{a t}$ is the atomic volume. $\mathcal{J}_{d}$ and $\mathcal{J}_{\alpha}$ correspond to fluxes of PDs and atoms respectively and can be expressed in terms of fluxes of atoms mediated by point defects, respectively $\mathcal{J}_{\alpha}^{\vee}$ for vacancy and $\mathcal{J}_{\alpha}^{\mathrm{l}}$ for interstitial mediated diffusion. We consider single vacancies and interstitials only. MD simulations [23,24] and experiments [25] have shown that PD clusters (especially interstitial ones) may be very mobile in pure iron. However, even under irradiation, their concentrations remain small when compared to the concentrations of single point defects. Moreover, some experiments [26] and MD simulations suggest that they are less mobile in $\mathrm{Fe}-\mathrm{Cr}$ alloys [27]. Therefore, the contribution of PD clusters is neglected in the present study.

The conservation principle implies that $\mathcal{J}_{d}$ and $\mathcal{J}_{\alpha}$ fluxes are simply a sum of $\mathcal{J}_{\alpha}^{d}$ fluxes:

$$
\begin{aligned}
& \mathcal{J}_{\alpha}=\sum_{d} \mathcal{J}_{\alpha}^{d} \\
& \mathcal{J}_{d}=\sum_{\alpha} \operatorname{sign}(d) \mathcal{J}_{\alpha}^{d}
\end{aligned}
$$

where $\operatorname{sign}(d)=-1$ for vacancies and $\operatorname{sign}(d)=1$ for interstitials. The fluxes $J_{\alpha}^{d}$ are expressed in the framework of the thermodynamics of irreversible processes [28]. In this formalism, the fluxes calcu- 
lated between neighboring PF cells are assumed to be a linear combination linking the chemical potential gradients of PDs and atoms, respectively $\nabla \mu_{d}$ and $\nabla \mu_{\beta}$, and the Onsager kinetic coefficients $L_{\alpha \beta}^{d}$ :

$\mathcal{J}_{\alpha}^{d}=-\sum_{\beta} L_{\alpha \beta}^{d}\left(\nabla \mu_{\beta}+\operatorname{sign}(d) \nabla \mu_{d}\right)$

The fluxes are thus expressed in terms of Onsager matrices of size $N_{a t} \times N_{a t}$ (with $N_{a t}$ the number of atomic species) dedicated to the diffusion of each PD, respectively $L_{\alpha \beta}^{V}$ for vacancy mediated diffusion and $L_{\alpha \beta}^{\mathrm{I}}$ for interstitial mediated diffusion, which correspond to macroscopic transport coefficients mediated by PDs for an isothermal system. For the sake of simplicity, we define the matrices of normalized Onsager coefficients as:

$\ell_{\alpha \beta}^{d}=\frac{V_{a t} k_{B} T}{X_{d}} L_{\alpha \beta}^{d}$

where $k_{B}$ is the Boltzmann constant and $T$ is the temperature of the system. Combining Eqs. (1)-(4), the diffusion equations can be expressed as follows:

$$
\begin{aligned}
& \frac{\partial X_{\alpha}}{\partial t}=\nabla \cdot\left[\sum_{d} \sum_{\beta} \frac{\ell_{\alpha \beta}^{d} X_{d}}{k_{B} T}\left(\nabla \mu_{\beta}+\operatorname{sign}(d) \nabla \mu_{d}\right)\right] \\
& \frac{\partial X_{d}}{\partial t}=\nabla \cdot\left[\sum_{\alpha} \sum_{\beta} \frac{\ell_{\alpha \beta}^{d} X_{d}}{k_{B} T}\left(\operatorname{sign}(d) \nabla \mu_{\beta}+\nabla \mu_{d}\right)\right]+K_{0}-K_{d}^{a b s}-K_{R}
\end{aligned}
$$

\subsubsection{Driving forces}

A classical Cahn-Hilliard approach introduces a single driving force, the alloy chemical potential gradient that is defined as the derivative of the alloy free energy with respect to the alloy composition. It is implicitly assumed that PDs are non-conservative species adapting their local concentration to reach the equilibrium PD concentration of a homogeneous alloy with a nominal composition equal to the local atomic concentration. In systems under irradiation, these PDs are not at local equilibrium and are treated here as conservative species. Therefore, in addition to the alloy driving force, PD driving forces are considered. Every PD chemical potential gradient is assumed to satisfy the Gibbs-Duhem relationship:

$\sum_{\beta}\left(X_{\beta} \nabla \mu_{\beta}+X_{d} \nabla \mu_{d}\right)=0$,

leading to driving forces expressed in terms of the alloy and PD driving forces. In order to maintain a general formulation, we consider the alloy A-B, where A refers to the solvent atom (here Fe atom) and $B$ refers to the solute atom (here $\mathrm{Cr}$ atom), leading to the relationships

$$
\begin{aligned}
& \nabla\left[\mu_{A}+\operatorname{sign}(d) \mu_{d}\right]=-\left(1-X_{A}\right) \nabla \mu+\operatorname{sign}(d) \nabla \mu_{d} \\
& \nabla\left[\mu_{B}+\operatorname{sign}(d) \mu_{d}\right]=\left(1-X_{B}\right) \nabla \mu+\operatorname{sign}(d) \nabla \mu_{d},
\end{aligned}
$$

where $\mu=\mu_{B}-\mu_{A}$ is the alloy chemical potential deduced from the alloy free energy and where it was assumed that $X_{d} \ll 1$. Here, we limit ourselves to the chemical contributions to both the alloy and PD chemical potentials. While long range elastic contributions associated with a redistribution of $\mathrm{Cr}$ atoms in Fe should be negligible due to the small size effect between $\mathrm{Fe}$ and $\mathrm{Cr}$, the elastic interactions between point defects and sinks are expected to produce a bias of elimination at sinks between vacancy and self-interstitials. However as a first step, the present study is focused on the estima- tion of radiation induced segregation profiles at neutral sinks and the elastic interactions are chosen to be neglected.

Both homogeneous and heterogeneous parts of the alloy chemical energy, $F_{\text {hom }}^{\text {chem }}$ and $F_{\text {het }}^{\text {chem }}$ respectively, are considered

$F^{\text {chem }}\left(X_{d}, X_{\alpha}\right)=F_{\text {hom }}^{\text {chem }}\left(X_{d}, X_{\alpha}\right)+F_{\text {het }}^{\text {chem }}\left(X_{\alpha}\right)$.

The homogeneous part of the alloy chemical energy depends on the activity coefficients of the alloying elements, $\gamma_{\alpha}$, and on the fraction of PDs at thermodynamic equilibrium conditions, $X_{d}^{e q}$, as follows

$F_{\text {hom }}^{\text {chem }}=\frac{k_{B} T}{V_{a t}}\left(\sum_{\alpha} \int_{\mathrm{V}} X_{\alpha} \ln \left(\gamma_{\alpha} X_{\alpha}\right) d V+\sum_{d} \int_{\mathrm{V}} X_{d} \ln \left(\frac{X_{d}}{X_{d}^{e q}}\right) d V\right)$.

$\alpha$ runs over the alloying elements and $d$ runs over the types of PD. Note that PD concentrations are assumed to be small so that interactions between PDs are neglected. The heterogeneous part of the alloy chemical energy depends on the stiffness parameter $\kappa$, and on the composition gradients as follows (the factor $1 / 4$ instead of $1 / 2$ is due to the summation over the chemical species):

$F_{\text {het }}^{\text {chem }}=\frac{1}{4 V_{a t}} \sum_{\alpha} \int_{\mathrm{V}} \kappa\left(\nabla X_{\alpha}\right)^{2} d V$

In the classical $\mathrm{CH}$ approach, $\kappa$ is considered as a purely thermodynamic quantity, corresponding to the energetic cost of concentration heterogeneities. The chemical potentials are thus obtained as:

$\mu=V_{a t} \frac{\delta F^{\text {chem }}}{\delta X_{\beta}}=V_{a t} \frac{\delta F_{\text {hom }}^{\text {chem }}}{\delta X_{\beta}}+V_{a t} \frac{\delta F_{\text {het }}^{\text {chem }}}{\delta X_{\beta}}=\mu^{\text {hom }}+\mu^{\text {het }}$
$\nabla \mu_{\beta}=\nabla\left[\mu_{\beta}^{\text {hom }}+\mu_{\beta}^{\text {het }}\right]=k_{B} T \Phi \nabla\left[\ln \left(X_{\beta}\right)\right]-\frac{\kappa^{\prime}}{X_{\beta}} \nabla^{3} X_{\beta}$

where $\Phi$ is the thermodynamic factor given by:

$\Phi=X_{A} X_{B} \frac{d \widetilde{\mu^{\text {hom }}}}{d X_{B}}$

with

$\widetilde{\mu^{\text {hom }}}=\frac{\mu^{\text {hom }}}{k_{B} T}$

and

$\kappa^{\prime}=X_{A} X_{B} \kappa$

A similar expression is obtained for $\nabla \mu_{\alpha}$.

However, it has been shown recently that kinetic correlations may depend on the spatial derivatives of the alloy driving force. A simple way to describe this phenomenon is to introduce an effective stiffness parameter $\kappa$ depending on both thermodynamic and kinetic parameters [19]. Direct AKMC simulations of nanoscale diffusion experiments were used to estimate the effective stiffness (Section 2.2.4) and therefore two parameters associated to the transport by vacancies $\kappa_{\mathrm{V}}$ and by interstitials $\kappa_{\mathrm{I}}$ will be considered in the following.

In the case of PD driving forces, as PD clustering is not considered, both thermodynamic and kinetic contributions to the effective stiffness parameters are neglected, leading to gradients of PD chemical potentials depending on the local concentration of both PD and chemical species through the variation of equilibrium PD fractions:

$\nabla \mu_{d}=\nabla\left[V_{a t} \frac{\delta F_{\text {hom }}^{\text {chem }}}{\delta X_{d}}\right]=\nabla\left[k_{B} T \ln \left(\frac{X_{d}}{X_{d}^{e q}}\right)\right]$.

By combining Eqs. (11) and (12), Eq. (5) can be rewritten using dimensionless units as follows: 


$$
\begin{aligned}
\frac{\partial X_{\alpha}}{\partial \tilde{t}}=\tilde{\nabla} \cdot\left[\sum_{d} \sum_{\beta} \tilde{\ell}_{\alpha \beta}^{d} X_{d}\left(\Phi \tilde{\mathbf{\nabla}} \ln \left(X_{\beta}\right)-\frac{\widetilde{\kappa_{d}^{\prime}}}{X_{\beta}} \tilde{\nabla}^{3} X_{\beta}+\operatorname{sign}(d) \tilde{\nabla} \ln \left(\frac{X_{d}}{X_{d}^{e q}}\right)\right)\right], \\
\frac{\partial X_{d}}{\partial \tilde{t}}=\tilde{\boldsymbol{\nabla}} \cdot\left[\sum _ { \alpha } \sum _ { \beta } \widetilde { \ell _ { \alpha \beta } ^ { d } } X _ { d } \left(\operatorname{sign}(d) \Phi \tilde{\nabla} \ln \left(X_{\beta}\right)-\operatorname{sign}(d) \frac{\widetilde{\kappa_{d}^{\prime}}}{X_{\beta}} \tilde{\nabla}^{3} X_{\beta}\right.\right. \\
\left.\left.+\tilde{\mathbf{\nabla}} \ln \left(\frac{X_{d}}{X_{d}^{e q}}\right)\right)\right]+\widetilde{K_{0}}-\widetilde{K_{d}^{a b s}}-\widetilde{K_{R}},
\end{aligned}
$$

where

$\tilde{t}=t / t_{0}$

$\widetilde{\ell_{\alpha \beta}^{d}}=\ell_{\alpha \beta}^{d} t_{0} / a_{0}^{2}$,

$\tilde{\nabla}=a_{0} \nabla$,

$\kappa_{d}^{\prime}=\kappa_{d} X_{A} X_{B} /\left(a_{0}^{2} k_{B} T\right)$,

$\widetilde{K_{0}}=K_{0} t_{0}$,

$\widetilde{K_{d}^{a b s}}=K_{d}^{a b s} t_{0}$

$\widetilde{K_{R}}=K_{R} t_{0}$,

where $t_{0}$ is a reference time which will be defined in Section 2.1.3 and $a_{0}$ is the grid spacing used in the PF simulation.

In Eq. (16), the recombination rate, $K_{R}$, is

$K_{R}(r, t)=R(r, t) X_{\mathrm{V}}(r, t) X_{\mathrm{I}}(r, t)$,

with the recombination term, $R$, given by:

$R(r, t)=4 \pi d_{r e c} \frac{D_{\mathrm{V}}(r, t)+D_{\mathrm{I}}(r, t)}{V_{a t}}$,

where $D_{\mathrm{V}}$ and $D_{\mathrm{I}}$ are the diffusion coefficients of PDs, and $d_{\text {rec }}$ the recombination distance between PDs. The diffusion coefficients of PDs can be calculated locally at each step of PF simulations knowing the diffusion matrices:

$D_{d}=\sum_{\alpha}^{N_{a t}} \sum_{\beta}^{N_{a t}} \ell_{\alpha \beta}^{d}$

Furthermore, $d_{\text {rec }}$ depends on irradiation conditions and has been set to $d_{\text {rec }}=0.994 \mathrm{~nm}$, which corresponds to the condition used in AKMC simulations of RIS, i.e. a PD recombination limited to fourth nearest neighbor distance.

In Eq. (16), the absorption rates of PDs $d=\mathrm{I}, \mathrm{V}$ at sinks, $K_{d}^{a b s}$, are defined as

$K_{d}^{a b s}(r, t)=\lambda(r) \lambda_{e f f}\left(X_{d}(r, t)-X_{d}^{S}\right)$

where $X_{d}^{S}$ is the PD site fraction at the surface of the sink depending on the local alloy concentration and $\lambda_{\text {eff }}$ corresponds to the sink efficiency. In this work, the composition inside the sink is maintained at $X_{d}^{S}$ by absorbing all the PDs in excess at each time step $d t$ of the PF simulation, i.e. by taking $\lambda_{\text {eff }}=1 / d t$. Two values of $X_{d}^{S}$ are considered. In a first set of calculations, we imposed a zero fraction of PDs at sink $\left(X_{d}^{S}=0\right)$ in order to be in the same simulation conditions as in AKMC simulations, thus allowing direct comparisons. In a second set of calculations, we imposed a thermal fraction of PDs at sinks $\left(X_{d}^{S}=X_{d}^{e q}(r, t)\right)$ in order to investigate their influence on RIS profiles. A planar sink is modeled by setting $\lambda=1$ in the sink and 0 otherwise.

\subsubsection{Numeric scheme and algorithm}

The PF method in this work is based on the previous method of Rouchette et al. [29-31], used to evaluate the sink strengths of dislocation loops interacting elastically with PDs. We use an explicit numerical scheme to solve Eq. (15). This means that the fractions of species at time $\tilde{t}+\widetilde{\delta} t$ are calculated from the fluxes at time $\tilde{t}$, where $\widetilde{\delta} t$ is the time increment used to solve the PF equations. At the beginning of each iteration, all the fields of the system are known at time $\tilde{t}$ in a grid of spacing $a_{0}$ formed by the discrete points $i$ located in the center of the PF cells represented in black in Fig. 1. The diffusion equations are spatially discretized using a finite difference scheme. The following operators are introduced: considering a dimensionless scalar field $\tilde{\varphi}, \widetilde{F D}+\left\{\tilde{\varphi}_{i}^{\tilde{t}}\right\}=\tilde{\varphi}_{i+1}^{\tilde{t}}-\tilde{\varphi}_{i}^{\tilde{t}}$ and $\widetilde{F D^{-}}\left\{\tilde{\varphi}_{i}^{\tilde{t}}\right\}=\tilde{\varphi}_{i}^{\tilde{t}}-\tilde{\varphi}_{i-1}^{\tilde{t}}$. We note $\left(\widetilde{\mathcal{J}}_{\alpha}\right)_{i}^{\tilde{t}}$ and $\left(\widetilde{\mathcal{J}}_{d}\right)_{i}^{\tilde{t}}$ the fluxes calculated at time $\tilde{t}$ in a shifted grid formed by the discrete points $i+1 / 2$ located at the boundaries of the PF cells represented in red in Fig. 1 and given by:

$$
\begin{aligned}
& \left(\widetilde{\mathcal{J}}_{\alpha}\right)_{i}^{\tilde{t}}=-\sum_{d} \sum_{\beta}\left(\widetilde{\ell_{\alpha \beta}^{d}}\right)_{i+\frac{1}{2}}^{\tilde{t}}\left(X_{d}\right)_{i+\frac{1}{2}}^{\tilde{t}}\left(\left(\tilde{\nabla} \widetilde{\mu}_{\beta}\right)_{i+\frac{1}{2}}^{\tilde{t}}+\operatorname{sign}(d)\left(\tilde{\mathbf{\nabla}} \widetilde{\mu}_{d}\right)_{i+\frac{1}{2}}^{\tilde{t}}\right) \\
& \left(\widetilde{\mathcal{J}}_{d}\right)_{i}^{\tilde{t}}=-\sum_{\alpha} \sum_{\beta}\left(\widetilde{\ell}_{\alpha \beta}^{d}\right)_{i+\frac{1}{2}}^{\tilde{t}}\left(X_{d}\right)_{i+\frac{1}{2}}^{\tilde{t}}\left(\operatorname{sign}(d)\left(\tilde{\mathbf{\nabla}} \widetilde{\mu}_{\beta}\right)_{i+\frac{1}{2}}^{\tilde{t}}+\left(\tilde{\nabla} \widetilde{\mu_{d}}\right)_{i+\frac{1}{2}}^{\tilde{t}}\right)
\end{aligned}
$$

where

$$
\begin{aligned}
& \left(X_{d}\right)_{i+\frac{1}{2}}^{\tilde{t}}=\left[\left(X_{d}\right)_{i+1}^{\tilde{t}}+\left(X_{d}\right)_{i}^{\tilde{t}}\right] / 2 \\
& \left(X_{\beta}\right)_{i+\frac{1}{2}}^{\tilde{t}}=\left[\left(X_{\beta}\right)_{i+1}^{\tilde{t}}+(X)_{i}^{\tilde{t}}\right] / 2 \\
& \left(\widetilde{\ell_{\alpha \beta}^{d}}\right)_{i+\frac{1}{2}}^{\tilde{t}}=\left[\left(\widetilde{\ell_{\alpha \beta}^{d}}\right)_{i+1}^{\tilde{t}}+\left(\widetilde{\ell_{\alpha \beta}^{d}}\right)_{i}^{\tilde{t}}\right] / 2 \\
& \Phi_{i+\frac{1}{2}}^{\tilde{t}}=\left[\Phi_{i+1}^{\tilde{t}}+\Phi_{i}^{\tilde{t}}\right] / 2 \\
& \left(\tilde{\nabla} \widetilde{\mu_{d}}\right)_{i+\frac{1}{2}}^{\tilde{t}}=F \tilde{D}^{+}\left\{\left(\widetilde{\mu_{d}}\right)_{i}^{\tilde{t}}\right\} \\
& \left(\tilde{\nabla} \widetilde{\mu_{\beta}}\right)_{i+\frac{1}{2}}^{\tilde{t}}=\Phi_{i+\frac{1}{2}}^{\tilde{t}} \widetilde{F D}^{+}\left\{\ln \left(X_{\beta}\right)_{i}^{\tilde{t}}\right\}-{\widetilde{\kappa_{d}}}_{d_{i+\frac{1}{2}}}^{\tilde{t}} /\left(X_{\beta}\right)_{i+\frac{1}{2}}^{\tilde{t}} \widetilde{F D}^{+}\left\{\widetilde{F D}^{-}\left\{F \tilde{D}^{+}\left\{\left(X_{\beta}\right)_{i}^{\tilde{t}}\right\}\right\}\right\} \\
& \widetilde{\kappa_{d}} \tilde{t}+\frac{1}{2}=\left[\widetilde{\kappa}_{d i+1}^{\tilde{t}}+\widetilde{\kappa_{d i}} \tilde{t}\right] / 2
\end{aligned}
$$

The fractions of species at time $\tilde{t}+\widetilde{\delta t}$ are then calculated as follows:

$$
\begin{aligned}
& \left(X_{\alpha}\right)_{i}^{\tilde{t}+\widetilde{\delta t}}=\left(X_{\alpha}\right)_{i}^{\tilde{t}}-\widetilde{\delta t} \widetilde{F D}^{-}\left\{(\widetilde{\mathcal{J}} \alpha)_{i}^{\tilde{t}}\right\} \\
& \left(X_{d}\right)_{i}^{\tilde{t}+\widetilde{\delta t}}=\left(X_{d}\right)_{i}^{\tilde{t}}-\widetilde{\delta t}\left[\widetilde{F D}^{-}\left\{\left(\widetilde{\mathcal{J}_{d}}\right)_{i}^{\tilde{t}}\right\}+\widetilde{K_{0}}-\left(\widetilde{K_{d}^{a b s}}\right)_{i}^{\tilde{t}}-\left(\widetilde{K_{R}}\right)_{i}^{\tilde{t}}\right]
\end{aligned}
$$

The set of diffusion Eq. (15) are highly stiff due to the significant difference of diffusion coefficients between species. In particular, interstitials are generally faster than vacancies, and both PDs diffuse much faster than atoms. In the explicit scheme, it is wellknown that the following stability criterion relating the physical time increment $\delta t$ and the diffusion coefficient $D$ of the migrating species must be fulfilled at each iteration and in each PF cell:

$\frac{\max _{i}\{D\} \delta t}{a_{0}^{2}}<\frac{1}{2 N_{\text {Dim }}}$

where $\max _{i}\{D\}$ is the spatial maximum of $D$ and $N_{\text {Dim }}$ is the dimensionality of the problem. $D=D_{d}$ for the PDs (Eq. (15)) and $D=D_{\alpha}$ for the chemical species $\alpha$, given by [2]:

$D_{\alpha}=\Phi \sum_{d}\left(\frac{\ell_{\alpha \alpha}^{d}}{X_{\alpha}}-\frac{\ell_{\alpha \beta}^{d}}{X_{\beta}}\right) X_{d}-\operatorname{sign}(d) \sum_{d}\left(\frac{\ell_{\alpha \alpha}^{d}+\ell_{\alpha \beta}^{d}}{X_{\alpha}}\right) X_{d} \xi_{d \alpha}$

with

$\xi_{d \alpha}=\frac{\partial \ln X_{d}^{e q}}{\partial \ln X_{\alpha}}$ 


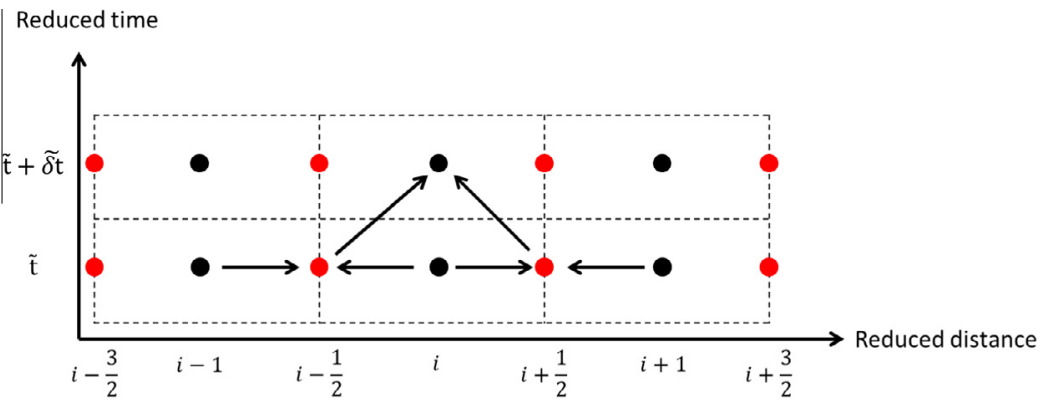

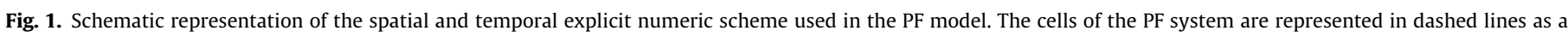

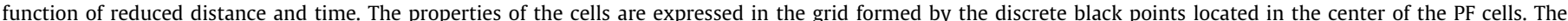

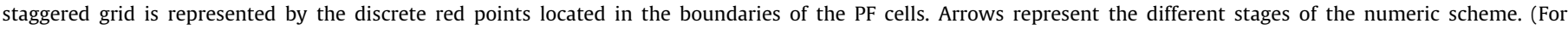
interpretation of the references to color in this figure legend, the reader is referred to the web version of this article.)

By choosing $t_{0}=a_{0}^{2} / \max _{i}\left\{D_{\mathrm{I}}\right\}$, used in Eq. (16), as the characteristic time for diffusion of interstitials and applying Eq. (24) to each species, it follows:

$$
\begin{aligned}
& \widetilde{\delta t_{\mathrm{I}}}<\frac{1}{2 N_{\text {Dim }}} \\
& \widetilde{\delta t_{\mathrm{V}}}<\frac{1}{2 N_{\text {Dim }}} \frac{\max _{i}\left\{D_{\mathrm{I}}\right\}}{\max _{i}\left\{D_{\mathrm{V}}\right\}} \\
& \widetilde{\delta t_{\alpha}}<\frac{1}{2 N_{\text {Dim }}} \frac{\max _{i}\left\{D_{\mathrm{I}}\right\}}{\max _{i, \alpha}\left\{D_{\alpha}\right\}}
\end{aligned}
$$

where $\widetilde{\delta t_{\mathrm{l}}}, \widetilde{\delta t_{\mathrm{V}}}$ and $\widetilde{\delta t_{\alpha}}$ are three distinct time steps associated respectively to the transport of interstitials, vacancies and atoms. With the numerical values of $D_{\mathrm{V}}, D_{\mathrm{I}}$ and $D_{\alpha}, \widetilde{\delta t_{\mathrm{I}}} \ll \widetilde{\delta t_{\mathrm{V}}} \ll \widetilde{\delta t_{\alpha}}$, which illustrates that several time scales are involved in this problem. For stability reasons, the use of only one time step would imply the choice of $\widetilde{\delta t_{1}}$, but a tremendous number of iterations would be required to reach the steady state, which makes this method not relevant.

Instead, we propose an algorithm in which all $\widetilde{\delta t_{\mathrm{l}}}, \widetilde{\delta t_{\mathrm{V}}}$ and $\widetilde{\delta t_{\alpha}}$ are used. One possible choice, adopted in the following, is to set $\widetilde{\delta t_{\mathrm{I}}}$ to a constant value $0.1, \widetilde{\delta t_{\mathrm{V}}}=\widetilde{\delta t_{\mathrm{I}}} \max _{i}\left\{D_{\mathrm{I}}\right\} / \max _{i}\left\{D_{\mathrm{V}}\right\}$ and $\widetilde{\delta t_{\alpha}}=\widetilde{\delta t_{\mathrm{I}}} \max _{i}\left\{D_{\mathrm{I}}\right\} / \max _{i, \alpha}\left\{D_{\alpha}\right\}$ to satisfy the stability criterions. Time integration can then be described by the following algorithm represented in Fig. 2: knowing all the properties of the system at time $\tilde{t}$, Eq. (3) is integrated with $\widetilde{\delta t_{\alpha}}$, allowing to obtain the field $X_{\alpha}$ at time $\tilde{t}+\widetilde{\delta} t_{\alpha}$. The second stage consists in computing the kinetic equations of PDs using the smaller time increments $\widetilde{\delta t_{\mathrm{I}}}$ and $\widetilde{\delta t_{\mathrm{V}}}$ as many times as needed to reach their quasi-steady states. The convergence criterion used is based on the fact that the fractions of PDs absorbed by the sink are equal to the fractions of PDs created in the system in this quasi-steady state:

$\left|1-\frac{\widetilde{K_{d}^{a b s}}}{\widetilde{K_{0}}}\right|<\varepsilon$

where $\varepsilon$ is a convergence parameter fixed at $10^{-2}$ in the following. By noting $n_{\mathrm{d}}$ the number of iterations required to reach this quasi-steady state, it is straightforward that $X_{d}^{\tilde{t}+\widetilde{\delta} t_{\alpha}}=X_{d}^{\tilde{t}+n_{d}} \widetilde{\delta t}_{d}$. It must be emphasized that the profile of the PD $d$ is in a quasi-steady state, since it is obtained for a given value of the field $X_{\alpha}^{\tilde{t}}$ which is not at its steady state and evolves much more slowly than PDs. After this second stage, all the fields $X_{\alpha}$ and $X_{d}$ are known at $\tilde{t}+\widetilde{\delta} t_{\alpha}$ and time integration can be performed on the following increment $\widetilde{\delta t_{\alpha}}$. Since $D_{\mathrm{V}}, D_{\mathrm{I}}$ and $D_{\alpha}$ are not constant, $\widetilde{\delta t_{\mathrm{V}}}$ and $\widetilde{\delta t_{\alpha}}$ are reevaluated at each time step. The resolution procedure is then repeated until the steady states of both atoms and PDs are reached.

In this multi-time step algorithm, the effective time step is $\widetilde{\delta t_{\alpha}}$ instead of $\widetilde{\delta t_{1}}$, which allows to reach the steady state of the system in realistic computational time, even if for each time step, a convergence procedure is required to reach the quasi-steady state of PDs. Practically, $n_{d} \sim 3$ except at the first time step for which $n_{d} \sim 10^{5}$. In all cases, $n_{d} \widetilde{\delta t_{d}} \ll \widetilde{\delta t_{\alpha}}$, which ensures that the algorithm is computationally efficient and keeps a physical meaning, it takes advan-

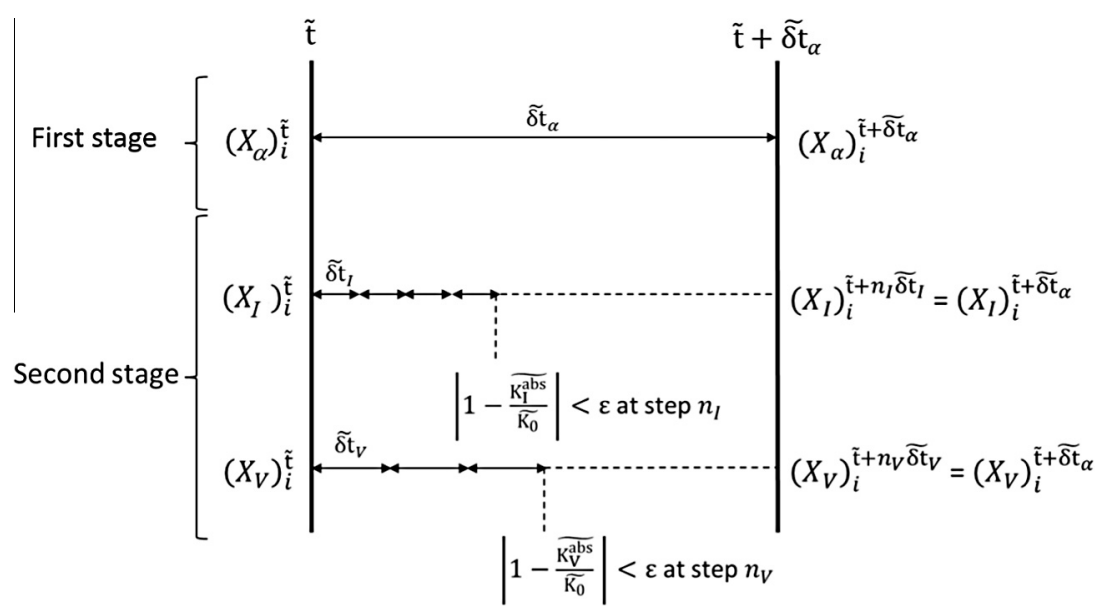

Fig. 2. Schematic representation of the multi-time step algorithm used in the PF model. 
tage of the fast achievement of PD quasi-steady state profiles during the calculations.

Note that at steady state, the total fluxes of vacancies and interstitials on a neutral PD sink are equal $\left(X_{\mathrm{I}} D_{\mathrm{I}}=X_{\mathrm{V}} D_{\mathrm{V}}\right)$. This is not the case during the transient regime, leading to a global shift of the sink, which can impact the transitional RIS profiles. A rough estimation leads to very small motions of the PD sink. In addition, recent numerical results [32] evaluate the maximum displacement of the sink to be of the order of $2 \times 10^{-3} \mathrm{~nm}$ (i.e. a small fraction of our grid spacing) using physical parameters similar to ours. For this reason the shift of the sink is neglected in this work.

\subsection{Monte Carlo modeling}

All the thermodynamic and kinetic parameters of Eq. (15) have been derived from Monte Carlo simulations based on the model and parameters of Ref. [13], using various equilibrium or kinetic methods. Here we only briefly recall of these methods, for the sake of clarity.

\subsubsection{Equilibrium PD concentrations}

Equilibrium PD concentrations are determined by the AKMC method presented in [33]. In simulations performed with one point defect (with $X_{d}^{M C}=1 / N_{a t}$ ), the relative PD concentrations in the various local environments $(i)$ are controlled by the local PD formation energies, i.e. the ratio $X_{d}^{M C}(i) / X_{d}^{e q}(i)$ is the same for each local environment. Therefore, the average PD concentration can be estimated, for example, by measuring the PD concentration $X_{d}^{M C}(\mathrm{Fe})$ on sites surrounded by pure iron. One gets, for the equilibrium concentration

$X_{d}^{e q}=X_{d}^{M C} \frac{X_{d}^{e q}(\mathrm{Fe})}{X_{d}^{M C}(\mathrm{Fe})}$

where $X_{d}^{e q}(\mathrm{Fe})=\exp \left(-G_{d}^{f o r}(\mathrm{Fe}) / k_{B} T\right), G_{d}^{\text {for }}(\mathrm{Fe})$ being the Gibbs free energy of PD in pure iron [13]. Simulations have been performed in systems on $16^{3}$ bcc sites, with $\mathrm{Cr}$ concentrations between $0 \%$ and $20 \%$ and temperatures between 600 and $1000 \mathrm{~K}$. As shown in
Figs. 3 and $4, X_{\mathrm{V}}^{e q}$ and $X_{\mathrm{I}}^{e q}$ are found to slightly depend on the composition.

\subsubsection{Transport coefficients}

AKMC simulations are used to calculate the normalized equilibrium phenomenological coefficients, $l_{\alpha \beta}^{d}$, dedicated to the diffusion of PDs. Starting from a thermodynamically converged configuration, one vacancy (respectively one interstitial) is introduced in the simulation box of $16^{3}$ bcc sites with periodic boundary conditions. Sites are therefore occupied by either Fe or $\mathrm{Cr}$ atoms except one site representing a PD. During the run, we calculate the statistical average of vector displacements of atoms $\alpha$ mediated by the $\mathrm{PD},\left\langle\Delta r_{\alpha}^{d}\right\rangle$, during the total Monte Carlo time, $t$. $l_{\alpha \beta}^{d}$ coefficients are then calculated using the generalized Einstein relations [4]:

$\ell_{\alpha \beta}^{d}=\frac{\left\langle\Delta r_{\alpha}^{d}\right\rangle \cdot\left\langle\Delta r_{\beta}^{d}\right\rangle}{6 t}$

Although this numerical method has proved its efficiency, it can be mentioned that its reliability strongly depends on the binding energies between chemical elements and PD by which diffusion occurs [34,35]. In case of important attraction, significant trapping and correlation effects occur (leading to the cancellation of an important number of atomic jumps), and large Monte Carlo steps (MCS) numbers are required. In order to insure the reliability of the calculation of Onsager coefficients, each atom in the simulation box must therefore achieve at least 10 jumps during the simulation. In this work, in order to reach satisfactory statistics, average values are calculated on $10^{5}$ series of $10^{5} \mathrm{MCS}$, and measurements are performed in homogeneous solid solutions, i.e. outside the miscibility gap or slightly inside (in the metastable state of the solid solution).

As explained in [13], we find that $l_{\alpha \mathrm{l}}^{\mathrm{I}}>0$ and $l_{\alpha \mathrm{V}}^{\mathrm{V}}<0$ in the whole range of composition and temperature investigated. Similar trends have been previously predicted by Wharry and coworkers (see [36] and references therein). This means that the fluxes of alloying elements are in opposite direction to the flux of $\mathrm{V}$ and in the same direction as the flux of SIA. Thus, we do not expect the

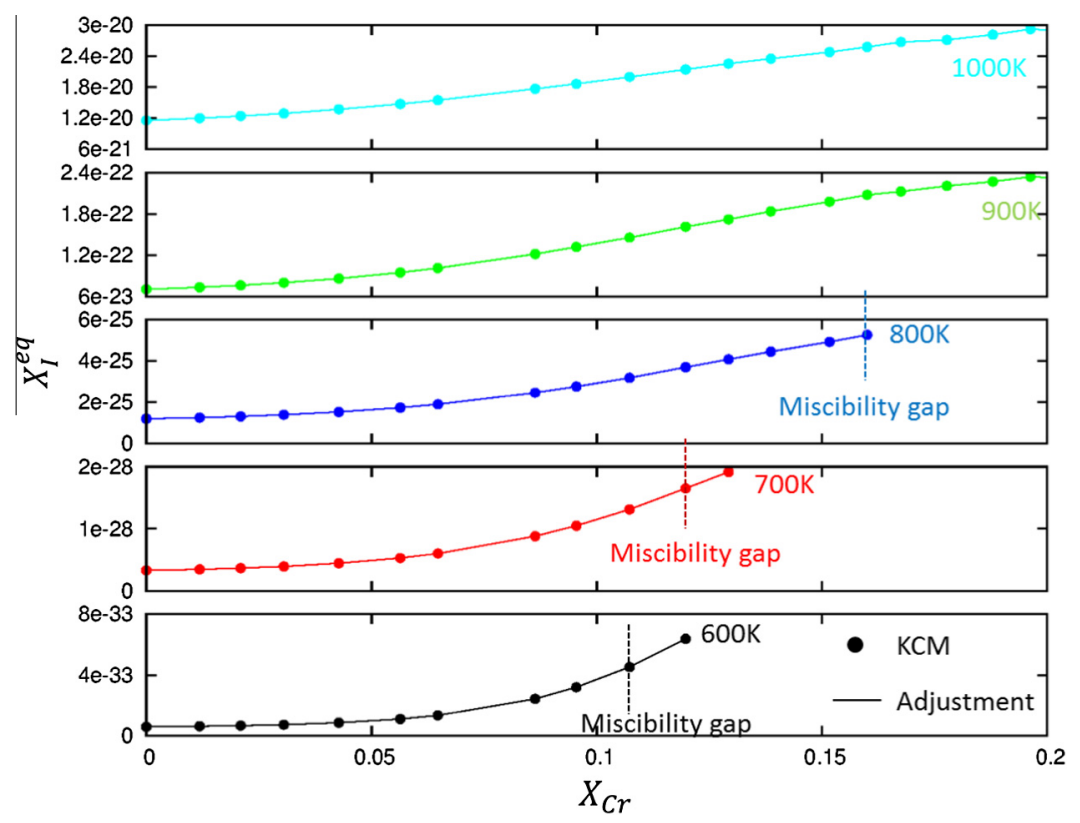

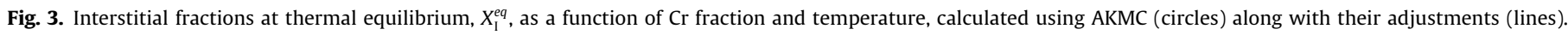
Vertical dashed lines represent the solubility limit (the miscibility gap). 


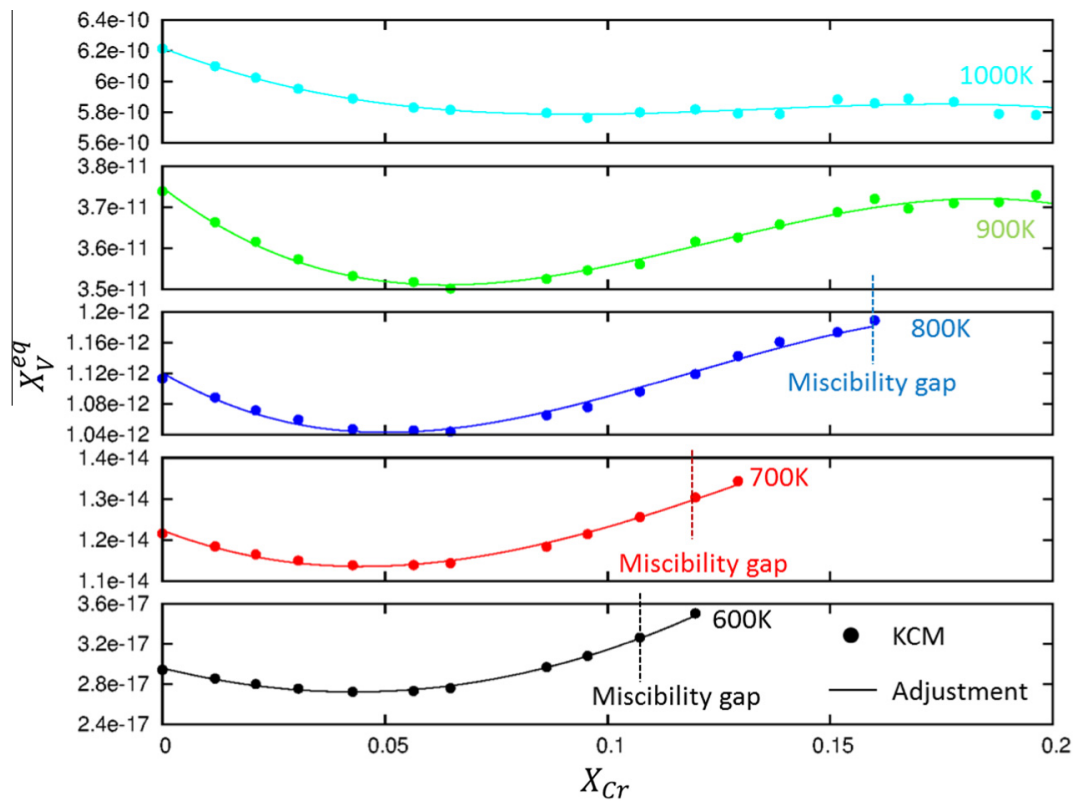

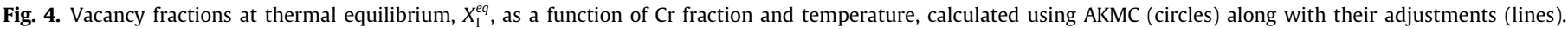
Vertical dashed lines represent the solubility limit (the miscibility gap).

solute drag mechanism to occur for the vacancy mediated diffusion. Note that this feature results from the point defects properties in $\mathrm{Fe}-\mathrm{Cr}$ alloys, where interactions between $\mathrm{Cr}$ atoms and vacancies are unusually weak $[11,13]$. In the other dilute $\mathrm{Fe}-\mathrm{X}$ alloys investigated, drag effects dominate at low temperatures and could be easily taken into account both in AKMC method and in PF models.

In steady-state conditions, fluxes are equal to zero and analytic models have been derived to determine steady-state concentration profiles (see Ref. [2] for a recent review). In simple cases (no precipitation, transport coefficients independent of the local composition), the segregation behavior of $\mathrm{Cr}$ atoms can be written in the binary $\mathrm{Fe}-\mathrm{Cr}$ in terms of the diffusion coefficients previously defined as follows:

$\frac{\nabla X_{\mathrm{Cr}}}{\nabla X_{\mathrm{V}}}=\frac{\ell_{\mathrm{FeV}}^{\mathrm{V}} \ell_{\mathrm{Fel}}^{\mathrm{I}}}{\left(\ell_{\mathrm{Fel}}^{\mathrm{I}} D_{\mathrm{Cr}}+\ell_{\mathrm{CrI}}^{\mathrm{I}} D_{\mathrm{Fe}}\right)}\left(\frac{\ell_{\mathrm{CrV}}^{\mathrm{V}}}{\ell_{\mathrm{FeV}}^{\mathrm{V}}}-\frac{\ell_{\mathrm{CrI}}^{\mathrm{I}}}{\ell_{\mathrm{Fel}}^{\mathrm{I}}}\right)$

$\mathrm{Cr}$ atoms tend to segregate at PD sinks when $\nabla X_{\mathrm{Cr}} / \nabla X_{\mathrm{V}}<0$, and deplete otherwise. Both $D_{\mathrm{Cr}}$ and $D_{\mathrm{Fe}}$ diffusion coefficients are positive outside the miscibility gap in the whole range of investigated temperatures. Thus, the sign of the $\mathrm{Cr}$ gradient is determined by the relative magnitude of the ratios $l_{\mathrm{CrV}}^{\mathrm{V}} / l_{\mathrm{FeV}}^{\mathrm{V}}$ and $l_{\mathrm{CrI}}^{\mathrm{I}} / l_{\mathrm{Fel}}^{\mathrm{I}}$ (given in Fig. 5). The vacancy contribution is dominant (i.e. $\mathrm{Cr}$ is expected to deplete near PD sinks) in a composition range that decreases with temperature. Above $900 \mathrm{~K}, \mathrm{Cr}$ is expected to deplete near sinks in the whole composition range investigated. At lower temperatures, the ratios $l_{\mathrm{CrV}}^{\mathrm{V}} / l_{\mathrm{FeV}}^{\mathrm{V}}$ and $l_{\mathrm{Crl}}^{\mathrm{I}} / l_{\mathrm{Fel}}^{\mathrm{I}}$ are equal at two crossover fractions between which $\mathrm{Cr}$ is expected to enrich, while outside of this composition range $\mathrm{Cr}$ is expected to deplete. The composition range between these crossover compositions increases with decreasing temperatures. These results are consistent with the previous experimental and modeling works of Wharry et al. [36,37].

A fifth-order polynomial function used to extrapolate the variation of the Onsager coefficients with composition (Fig. 5) will be used as input parameters of the PF method.

\subsubsection{Thermodynamic factor}

The thermodynamic factor $\Phi$ is related to the change of the alloy chemical potential difference with respect to the alloy com- position. In dilute alloys, $\Phi=1$ since every solute atom has the same pure local environment. In concentrated alloys, $\Phi$ is positive when the stable state is a homogeneous solid solution and a twophase infinite system would give a thermodynamic factor equal to zero. The variation of the alloy chemical potential is computed by performing Monte Carlo Metropolis (MCM) simulations combined with a Widom integration as explained in [20]. To do so, a first run is performed to reach equilibrium, then virtual exchanges between alloying elements are performed after every series of $10^{5}$ MCS (1 MCS corresponding to an exchange trial between $1 \mathrm{nn}$ atoms). The simulation box contains $32^{3}$ bcc sites occupied by either Fe or $\mathrm{Cr}$ atoms, with periodic boundary conditions. The energy $\Delta E^{\alpha \rightarrow \beta}$ related to the exchange of a single atom of species $\alpha$ by an atom of species $\beta$ is calculated for the $N_{\alpha}$ atoms of species $\alpha$. After $N$ series, the alloy chemical potential is computed from the statistical average

$\tilde{\mu}=\frac{1}{N_{\alpha} N} \sum_{i=1}^{N} \ln \left(\sum_{j \in \alpha} \exp \left(-\frac{\Delta E^{\alpha \rightarrow \beta}}{k_{B} T}\right)\right)$

This method can be applied to stable solid solutions, but also to metastable ones where MCM measurements of chemical potential can be performed before $\alpha^{\prime}$ precipitation starts. The thermodynamic factor $\Phi$, derived from the alloy chemical potential and Eq. (12) is shown in Fig. 6 with respect to $\mathrm{Cr}$ concentration up to 20 at.\% and temperatures between 600 and $1000 \mathrm{~K}$. $\Phi$ first increases and then decreases with increasing $\mathrm{Cr}$ concentration. The lower the temperature, the greater is the variation of $\Phi$ with composition. Inside the $\alpha-\alpha^{\prime}$ miscibility gap associated with the Monte Carlo model used in this work [13,38], $\Phi$ is found to become negative below $900 \mathrm{~K}$, meaning that the solid solution is unstable. However, the MCM calculations associated with negative values of $\Phi$ cannot be trusted due to the possible $\alpha-\alpha^{\prime}$ decomposition [20] and will not be considered in this work.

\subsubsection{Effective stiffness parameters}

To estimate the stiffness parameters, a periodic simulation box is built from a bcc lattice including a total number of sites equal to $512^{3} / 4$. Sites are occupied by either Fe or $\mathrm{Cr}$ atoms except one site representing a PD. Following the procedure explained in [19], the 


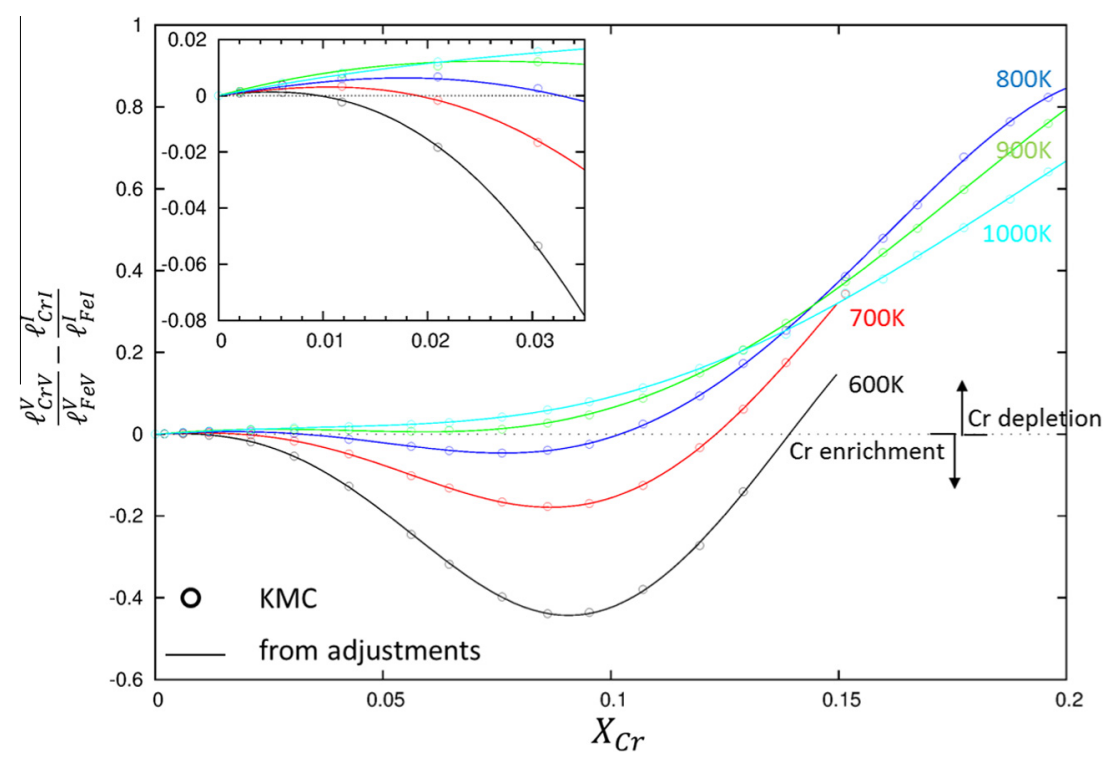

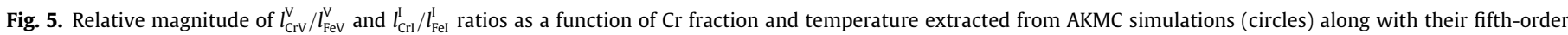
polynomial adjustments (lines).

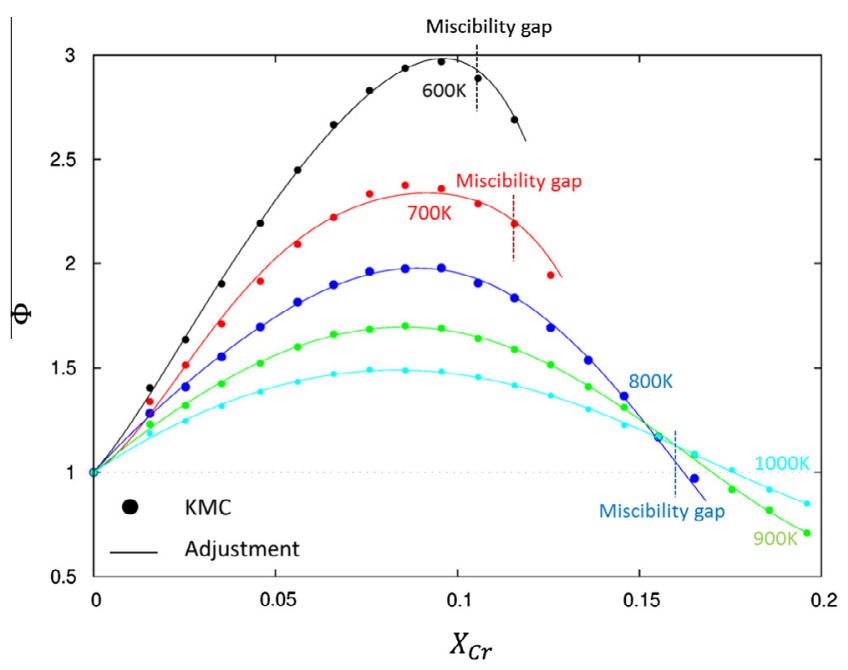

Fig. 6. Thermodynamic factor, $\Phi$, as a function of $\mathrm{Cr}$ fraction and temperature, measured in MCM simulations (circles) along with their adjustments (solid lines).

initial composition field of species $\alpha, X_{\alpha}$, of every simulation is a 1D sinusoidal composition-modulated solid solution along a [100] direction of the bcc lattice with an amplitude $\delta X_{\alpha}^{\max }=0.01$ and a wavelength $\lambda$. The solid solution is then annealed, the concentration fluctuation decaying and the system evolving toward its equilibrium state. The temperature is chosen to be above the critical temperature so that the equilibrium state corresponds to a uniform solid solution. At a given time, the sinusoidal average amplitude $\delta X_{\alpha}$ of species $\alpha$ is obtained from the $\lambda$ component of the Fourier transformed 1D concentration field. In the AKMC simulations, the defect is considered as a conservative species. The effective interdiffusion coefficient $D_{d}^{\text {int }}$ is extracted from the time variation of the logarithm of $\delta X_{\alpha}$. A linear behavior is observed and the slope is equal to $k^{2} D_{d}^{i n t}$ for small $k$ [19]:

$\ln \left(\delta X_{\alpha} / \delta X_{\alpha}^{\max }\right)=-k^{2} D_{d}^{\text {int }} t$

The stiffness parameter $\kappa_{d}$ can be hence extracted from a fit of $D_{d}^{\text {int }}$ versus $k^{2}$ assuming a linear behavior at small $k$,
$D_{d}^{i n t}=D_{d}^{\text {int }}(k=0)\left[1+\frac{\kappa_{d} X_{A} X_{B}}{k_{B} T \Phi} k^{2}\right]$

$\kappa_{d}$ is thus extracted from the variation of $D_{d}^{\text {int }}$ between the wavevectors $k=2 \pi /(8 a)$ and $k=2 \pi /(4 a)$, $a$ being the lattice parameter. The standard error on $\kappa_{d}$ is estimated to be smaller than $10 \%$.

As shown in [19], $\kappa$ includes kinetic contributions and depends on the PD diffusion mechanism. The measured values of $\kappa$ and the corresponding polynomial fit for the vacancy and interstitial diffusion mechanism are plotted in Fig. 7.

Within the ranges of temperature and composition investigated in this work, the calculated values of $\kappa_{d}$ do not have a significant influence on the segregation behavior. However $\kappa_{d}$ will have a strong influence on situations where phase separation occurs. Those will be addressed in future developments.

\subsubsection{Radiation induced segregation simulations}

Simulations of RIS are performed in boxes of $512 \times 64 \times 64$ bcc sites, with a planar PD sink located in the middle of the long dimension of size $e=100 \mathrm{~nm}$. Sites are occupied by either Fe or

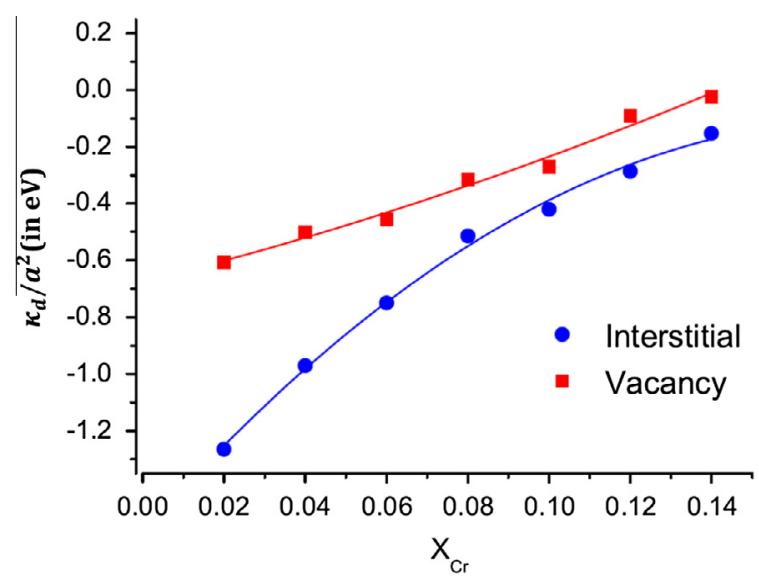

Fig. 7. Stiffness parameter $\kappa_{\mathrm{V}} / a^{2}$ and $\kappa_{\mathrm{I}} / a^{2}$ in eV as a function of $\mathrm{Cr}$ fraction at temperature $T=700 \mathrm{~K}$ interpolated by a second order polynomial, extracted from AKMC simulations of a composition-modulated solid solution along a [100] direction for the vacancy and the interstitial diffusion mechanism. 

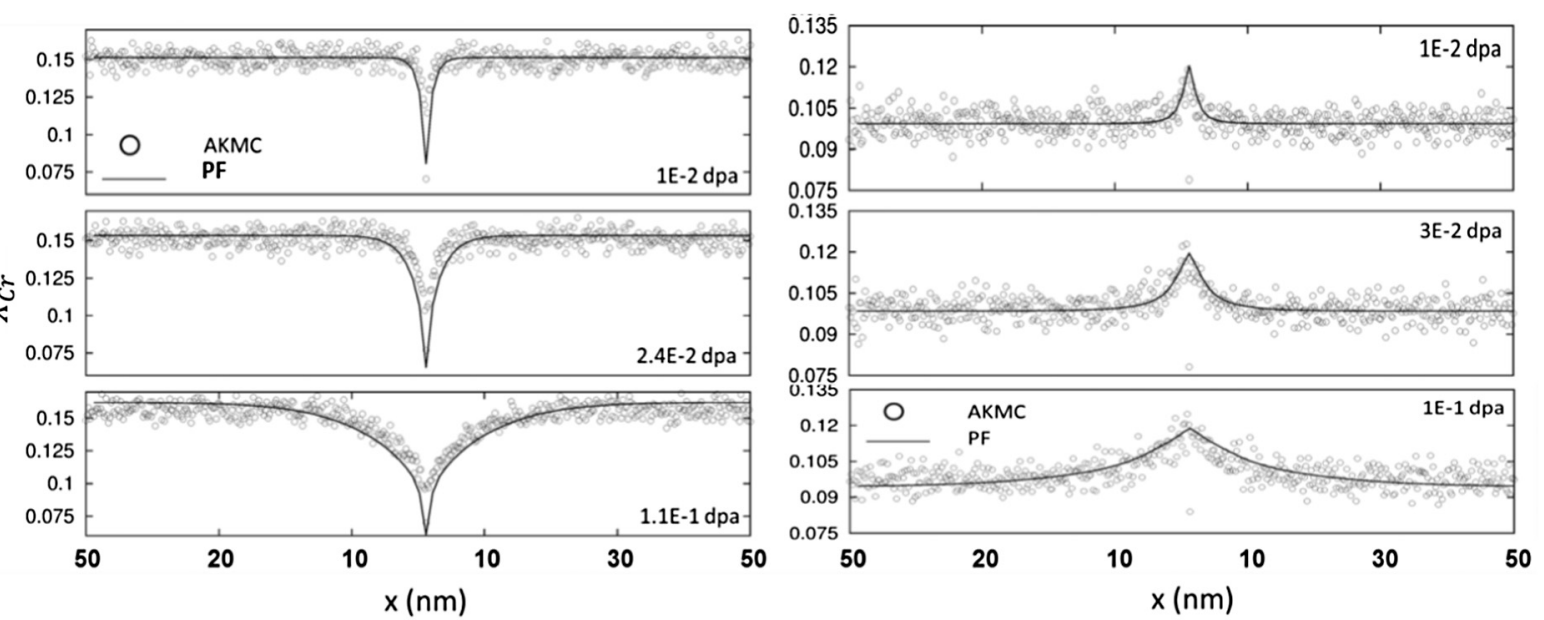

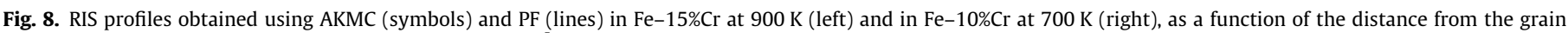
boundary, $x$, and for different irradiation doses at $K_{0}=10^{-5} \mathrm{dpa} \mathrm{s}^{-1}$.

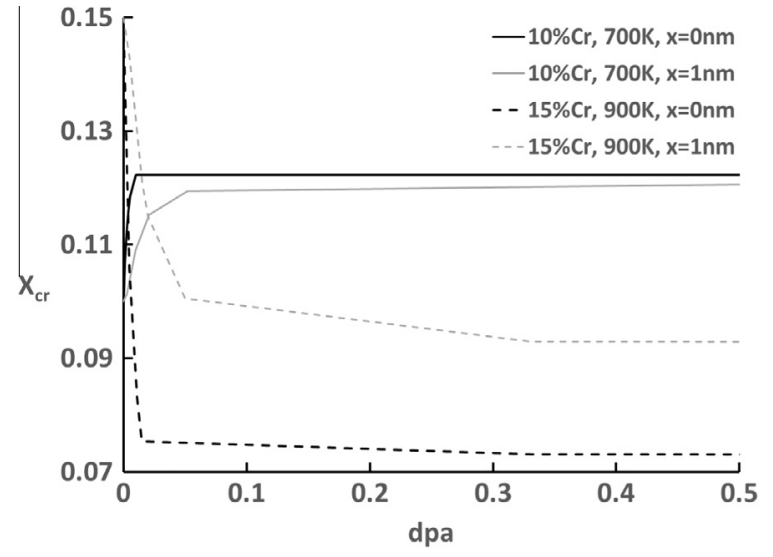

Fig. 9. Concentration of $\mathrm{Cr}$ at $\operatorname{sink}(x=0 \mathrm{~nm}$, black line) and at a distance of $1 \mathrm{~nm}$ from the $\operatorname{sink}(x=1 \mathrm{~nm}$, grey line) as a function of dose in $\mathrm{Fe}-15 \% \mathrm{Cr}$ at $900 \mathrm{~K}$ (dotted line) and in $\mathrm{Fe}-10 \% \mathrm{Cr}$ at $700 \mathrm{~K}$ (full line), calculated using PF.
Cr or a PD (I or V). These simulations take into account the same events as in the PF modeling [13]:

(1) The creation of Frenkel pairs with a production rate $K_{0}$. PD pairs are randomly created within the simulation box, along sequences of 10 replacements in $\langle 111\rangle$ directions. These events limited to the creation of single PDs simulate electron irradiation rather than neutron or heavy ion irradiation.

(2) The migration of interstitials and vacancies, according to the diffusion model.

(3) The recombination of PD pairs below a recombination radius to fourth nearest neighbors.

(4) The annihilation of PDs at sinks.

\section{Validation of the PF model against AKMC and rate theory}

In order to validate the PF model, direct comparisons of RIS profiles obtained from PF and AKMC at various temperatures, nominal alloy compositions and PD generation rates have been achieved. Since AKMC simulations do not take into account the creation of

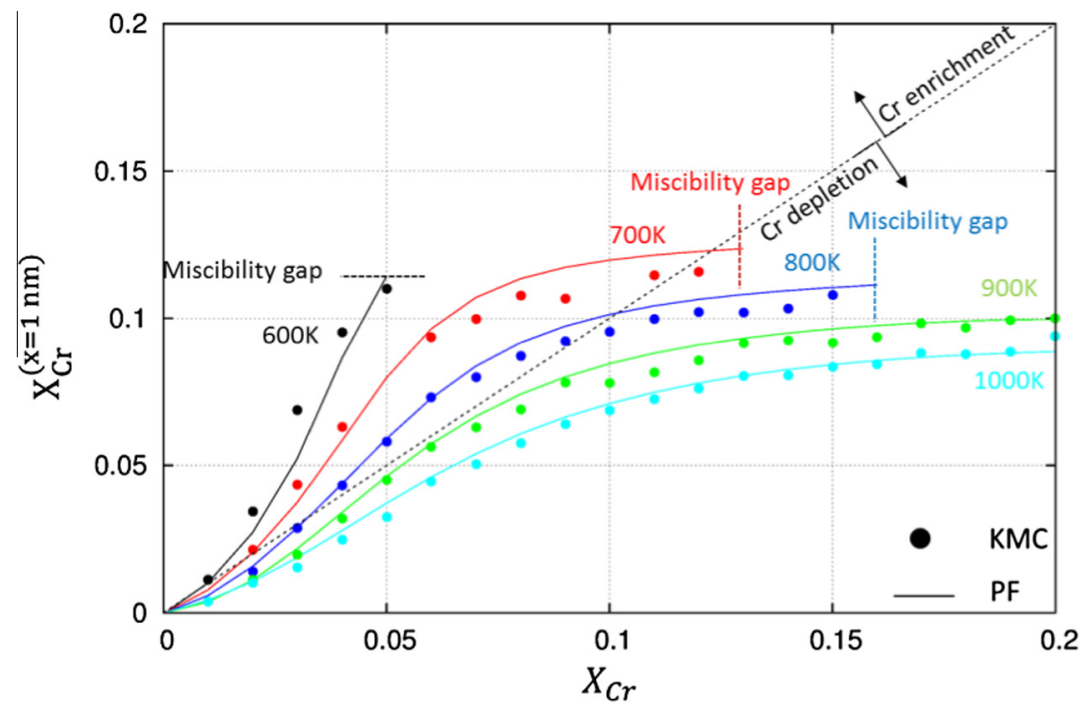

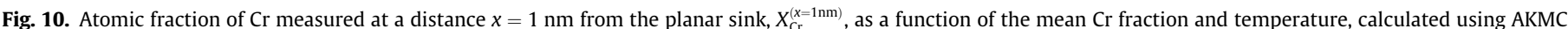

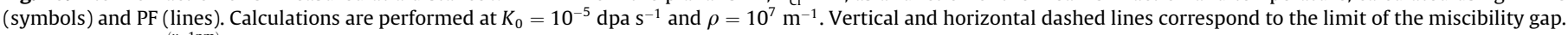
The dashed line $X_{\mathrm{Cr}}^{(x=1 \mathrm{~nm})}=X_{\mathrm{Cr}}$ represents the limit between $\mathrm{Cr}$ depletion and $\mathrm{Cr}$ enrichment. 

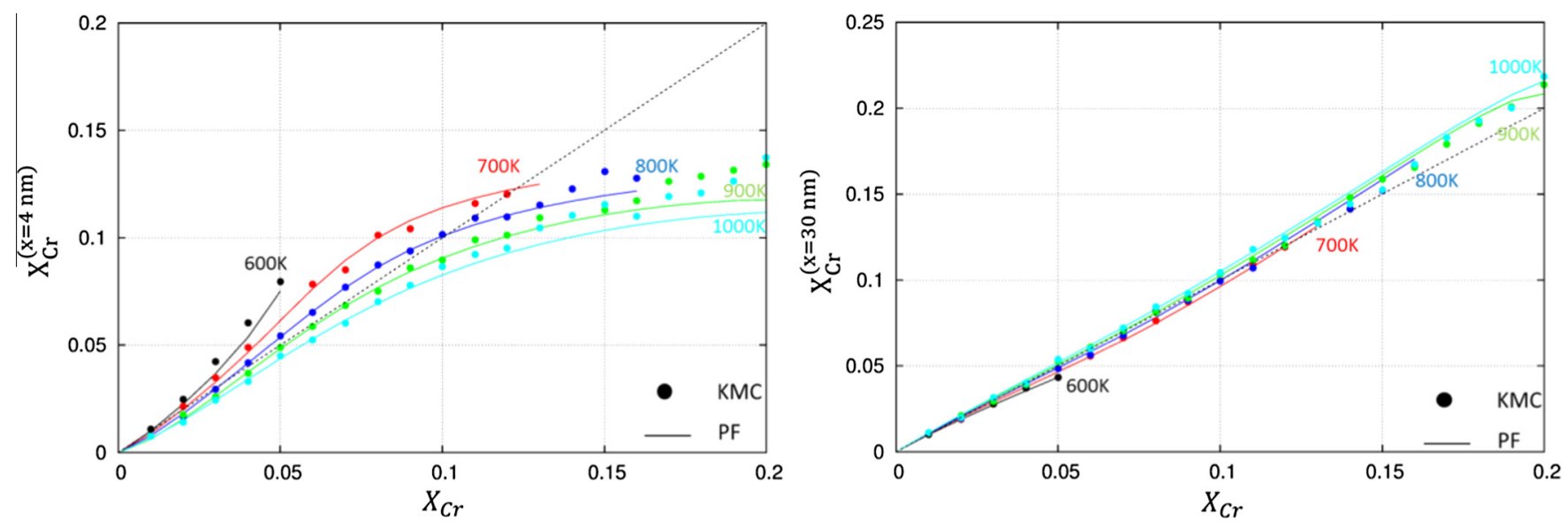

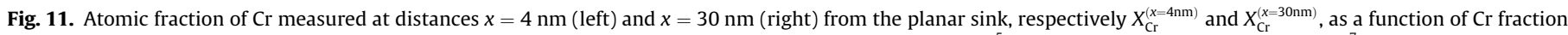

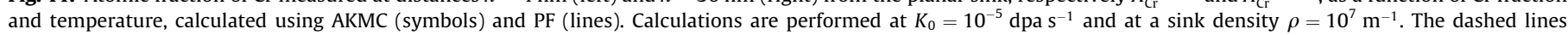
represent the limit between $\mathrm{Cr}$ depletion and $\mathrm{Cr}$ enrichment.

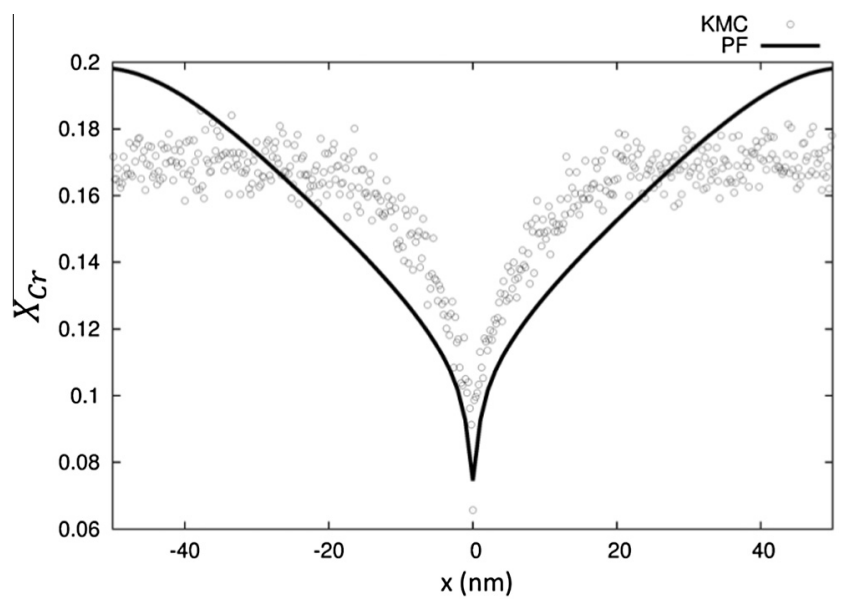

Fig. 12. RIS steady-state profiles obtained using AKMC (symbols) and using PF (lines) in $\mathrm{Fe}-16 \% \mathrm{Cr}$ at $900 \mathrm{~K}$, as a function of the distance from the grain boundary, $x$, at $K_{0}=10^{-5}$ dpa s$^{-1}$.

equilibrium $\mathrm{PD}$ at sinks, in order to allow direct comparisons between PF and AKMC, a zero fraction of PDs at the sink $\left(X_{d}^{S} \approx 0\right)$ is imposed in the PF simulations of this section. In prac- tice, in order to avoid numerical instabilities, $X_{d}^{S}$ is put equal to a very small value well below $X_{\mathrm{V}}^{e q}$. The one dimensional PF simulations were done in a system containing 100 cells of size $a_{0}=1 \mathrm{~nm}$, with a planar sink of width $a_{0}$, representing a grain boundary (GB).

A satisfying agreement between AKMC and PF simulations of the kinetics of RIS profiles is obtained, in the whole range of temperatures, compositions, and PD generation rates investigated in this study. As an illustration, Fig. 8 represents the RIS profiles obtained by AKMC and PF for two different compositions and temperatures. Within the temperature range of $\mathrm{Cr}$ enrichment, the agreement is better. The $\mathrm{Cr}$ concentration very rapidly reaches its steady-state value at the GB (at an irradiation dose roughly equal to $10^{-2} \mathrm{dpa}$, see Fig. 9), and the whole concentration profile is stable after typically a few 0.1 dpa (see also Fig. 6 in Ref. [13]). Experiments usually show a slower evolution and reach steady state up to doses of several dpa [36]: the present results suggest that the kinetics of RIS at a stationary sink is indeed very rapid and that the experimental delay is due to the evolution of the PD sink microstructure.

A systematic study of segregation at a distance $x=1 \mathrm{~nm}$ close to the planar sink, $X_{\mathrm{Cr}}^{(x=1 \mathrm{~nm})}$, is presented in Fig. 10. A good agreement between $\mathrm{PF}$ and $\mathrm{AKMC}$ is obtained although $\mathrm{Cr}$ segregation at GB predicted by the PF method is in most cases above the AKMC
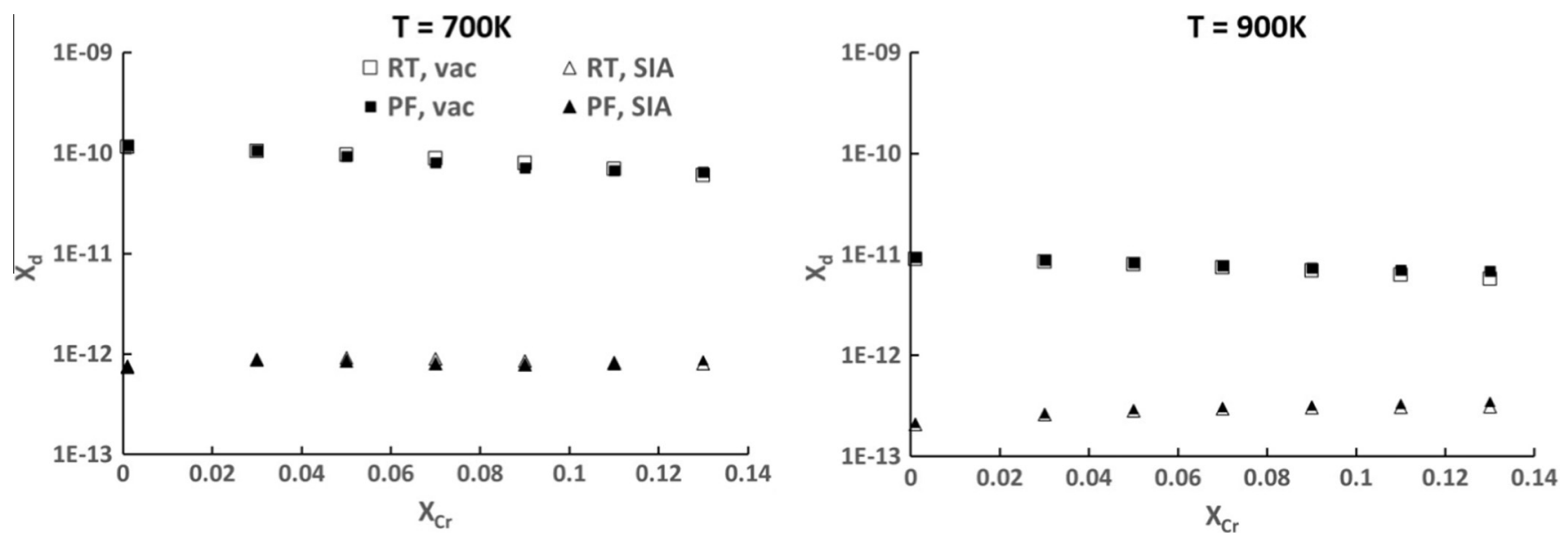

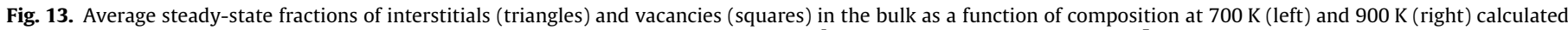
using PF (filled symbols) and rate theory (open symbols) for an irradiation rate $K_{0}=10^{-5} \mathrm{dpa} \mathrm{s}^{-1}$ and a sink density $\rho=10^{7} \mathrm{~m}^{-1}$. 

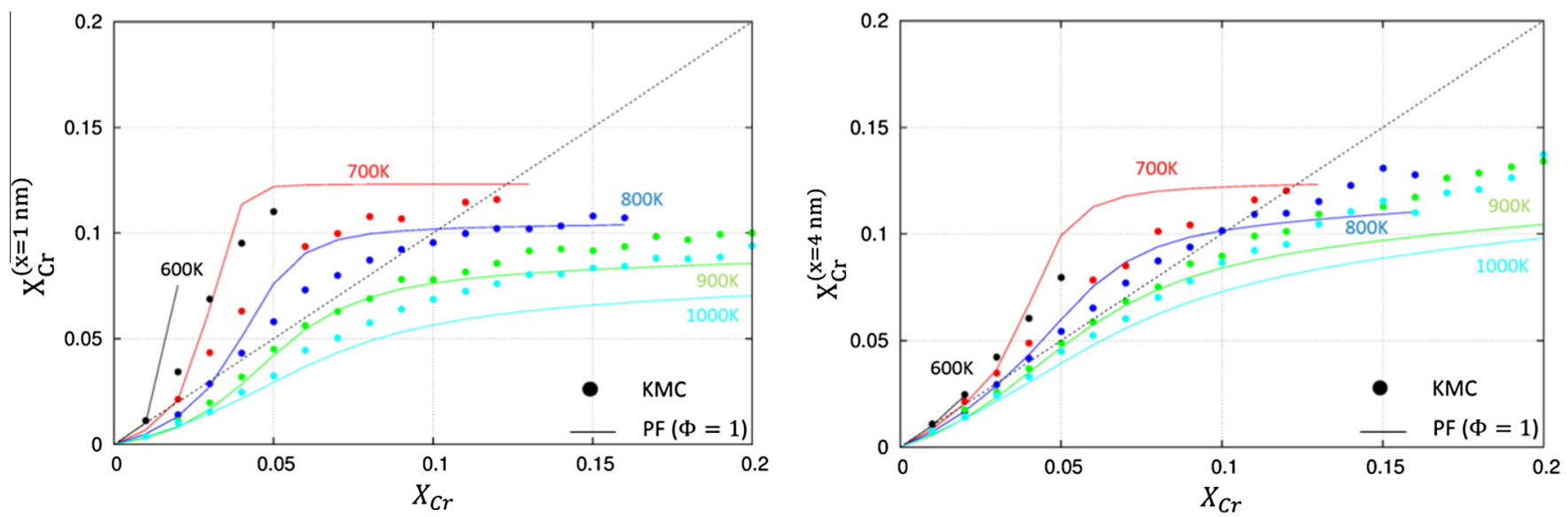

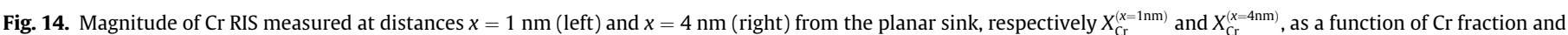

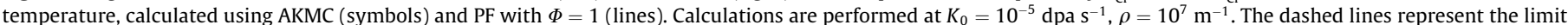
between $\mathrm{Cr}$ depletion and $\mathrm{Cr}$ enrichment.

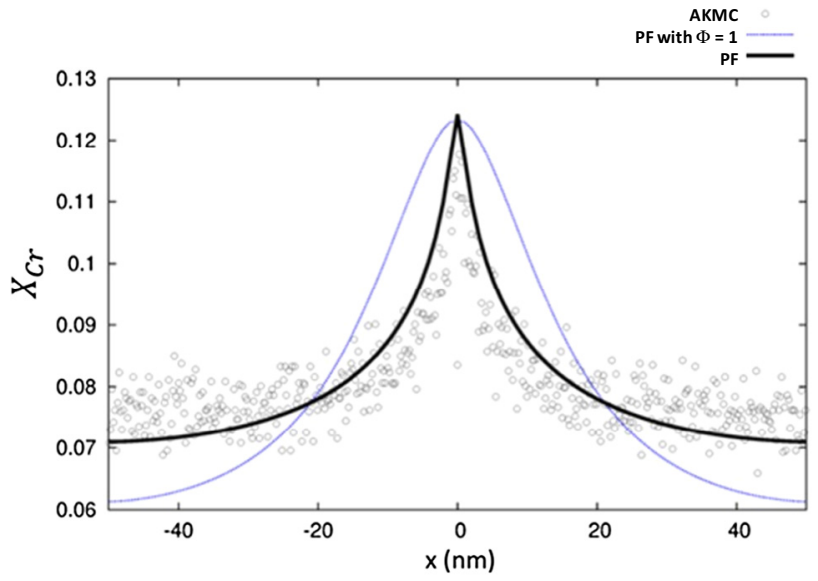

Fig. 15. RIS steady-state profiles obtained using AKMC (symbols), PF with adjustments of $\Phi$ obtained in Fig. 6 (thick line), and PF with $\Phi=1$ (thin line) in Fe-8\%Cr at $700 \mathrm{~K}$, as a function of the distance from the grain boundary, $x$, at $K_{0}=10^{-5} \mathrm{dpa} \mathrm{s}^{-1}$.

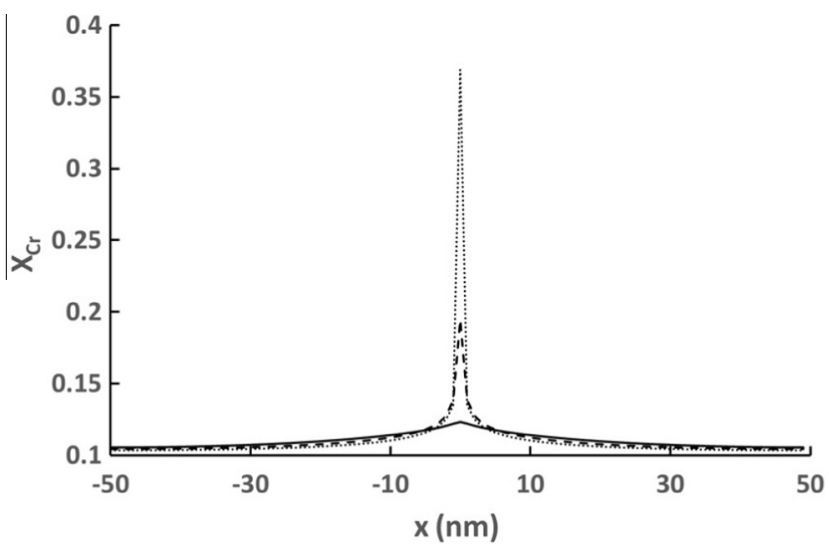

Fig. 16. $X_{\mathrm{Cr}}$ at the steady state around the sink for an alloy with $11 \% \mathrm{Cr}$ at $700 \mathrm{~K}$ for 3 distinct sets of modeling parameters (see text for details).

values. It can also be seen that the trends of RIS behavior predicted in Fig. 5 are reproduced in Fig. 10. Indeed, above $900 \mathrm{~K}$, the sink tends to deplete in $\mathrm{Cr}$ within the whole investigated composition range. Furthermore, $\mathrm{Cr}$ depletion intensity is less important for $X_{\mathrm{Cr}}<0.1$, corresponding to compositions at which
$l_{\mathrm{CrV}}^{\mathrm{V}} / l_{\mathrm{FeV}}^{\mathrm{V}}-l_{\mathrm{Crl}}^{\mathrm{l}} / l_{\mathrm{Fel}}^{\mathrm{I}}$ values are the smallest. At lower temperatures, RIS is suppressed at crossover fractions for which $l_{\mathrm{CrV}}^{\mathrm{V}} / l_{\mathrm{FeV}}^{\mathrm{V}}=l_{\mathrm{Crl}}^{\mathrm{I}} / l_{\mathrm{Fel}}^{\mathrm{I}}$.

For the purpose of comparison of RIS profiles, the composition dependence of $\mathrm{Cr}$ segregation at distances $x=4 \mathrm{~nm}$ and $x=30 \mathrm{~nm}$ from the planar sink is represented in Fig. 11. A good agreement between PF and AKMC results is obtained except at $\mathrm{Cr}$ composition above 15 at.\%. Indeed, we can already see in Fig. 8 that a better agreement is obtained at 10 at.\% $\mathrm{Cr}$ than at 15 at.\% $\mathrm{Cr}$. This issue is better illustrated in Fig. 12 where we present a direct comparison of RIS steady-state profiles between PF and AKMC at 16 at.\% $\mathrm{Cr}$ and $900 \mathrm{~K}$. Note that due to the small size of the MC system, the strong depletion of $\mathrm{Cr}$ near the sink leads to $\mathrm{Cr}$ concentration above the nominal value even at the maximum distance $(\sim 50 \mathrm{~nm})$, the effect is even more pronounced in the PF model because the width of the depletion profile is overestimated. The less satisfying agreement of the RIS profiles between PF and AKMC observed in concentrated $\mathrm{Cr}$ solid solutions may be due to the difficulty of measuring the thermodynamic factor close to the miscibility gap.

The average steady-state fractions of PDs in the bulk of irradiated $\mathrm{FeCr}$ alloys obtained from PF simulations have also been compared to the analytical solutions $X_{\mathrm{V}}^{R T}$ and $X_{\mathrm{I}}^{R T}$ deduced from rate theory equations, assuming no effect of RIS on the steady-state PD fractions:

$X_{\mathrm{V}}^{R T}=-\frac{K_{\mathrm{I}}}{2 R}+\sqrt{\left(\frac{K_{\mathrm{I}}}{2 R}\right)^{2}+\frac{K_{0} K_{\mathrm{I}}}{R K_{\mathrm{V}}}}$
$X_{\mathrm{I}}^{R T}=-\frac{K_{\mathrm{V}}}{2 R}+\sqrt{\left(\frac{K_{\mathrm{V}}}{2 R}\right)^{2}+\frac{K_{0} K_{\mathrm{V}}}{R K_{\mathrm{I}}}}$

In which $K_{d}=D_{d} k_{d s}^{2}$ and $k_{d s}^{2}$ is the sink strength, which in the case of a planar sink is equal to $12 / \mathrm{e}^{2} . D_{\mathrm{I}}$ and $D_{\mathrm{V}}$ have been estimated from Eq. (19) using the values of $l_{\alpha \beta}^{d}$ corresponding to the nominal compositions. The results are in excellent agreement for both investigated temperatures in the whole composition range as presented in Fig. 13, for interstitials and for vacancies.

\section{Discussion: influence of the physical parameters on RIS profiles}

The previous results have been obtained by fully taking into account the variation of thermodynamic and kinetics parameters with the local composition of the alloy. In order to emphasize the importance of these variations, we will present PF results 
obtained with constant and uniform values of the same parameters.

\subsection{Effect of the variation of the thermodynamic factor}

The solid solution approximation $(\Phi=1)$ is generally considered in previous RIS models (see for instance [27]). However, this parameter is found to have a significant impact on the shape of the RIS profiles. The effect of $\Phi$ is illustrated in Figs. 14 and 15. In Fig. 14, the agreement is not as good as in Figs. 10 and 11. Fig. 15 provides a direct comparison between AKMC and PF segregation profiles. A good agreement is obtained when the AKMC value of $\Phi$ is used as input of the PF method while a large discrepancy is observed with $\Phi=1$. The latter approximation leads to PF segregation profiles broader than the AKMC ones. The strong effect of $\Phi$ on RIS highlights the role of backward atomic diffusion acting against the segregation profile produced by the flux coupling.

\subsection{Effect of the variation of the transport coefficients}

The importance of taking into account the correct composition dependence of the transport coefficients to obtain quantitative results is illustrated in Fig. 16. This figure presents the results of RIS for an alloy containing $11 \% \mathrm{Cr}$ at $700 \mathrm{~K}$. Three modeling conditions were adopted: i. The solid line corresponds to a simulation where the dependence of $\Phi, X_{d}^{e q}$ and $l_{\alpha \beta}^{d}$ on the local composition is taken into account. As already predicted by Fig. 5, there is a local $\mathrm{Cr}$ enrichment at the sink.

ii. The dashed line corresponds to a simulation done with constant values of $\Phi, X_{d}^{e q}$ and $l_{\alpha \beta}^{d}$ at the nominal alloy composition. As a result, the segregation is enhanced compared to the previous case. This can be easily understood since according to Fig. 5, at $700 \mathrm{~K}$ an increase of the composition above $11 \% \mathrm{Cr}$ leads to a decrease of the segregation tendency and even to an inversion of the flux coupling above $12.5 \% \mathrm{Cr}$. Therefore, the $\mathrm{Cr}$ enrichment is strongly decreased when the variation of the flux coupling with the local concentration is accounted for.

iii. The dotted line corresponds to a simulation taking into account the variation of $\Phi$ and $X_{d}^{e q}$, whereas $l_{\alpha \beta}^{d}$ are assumed to be fixed at the nominal composition. As a result, the segregation is enhanced and in fact the composition at the sink diverges (i.e. does not reach a steady-state value). This is due to the fact that the polynomial fit used to reproduce the $\Phi$ composition dependence in Fig. 6 is not valid for values above $15 \% \mathrm{Cr}$ (in fact it gives $\Phi=-200$ for $30 \% \mathrm{Cr}$ ) and produces an artificial spinodal decomposition in the neighborhood of the sink.

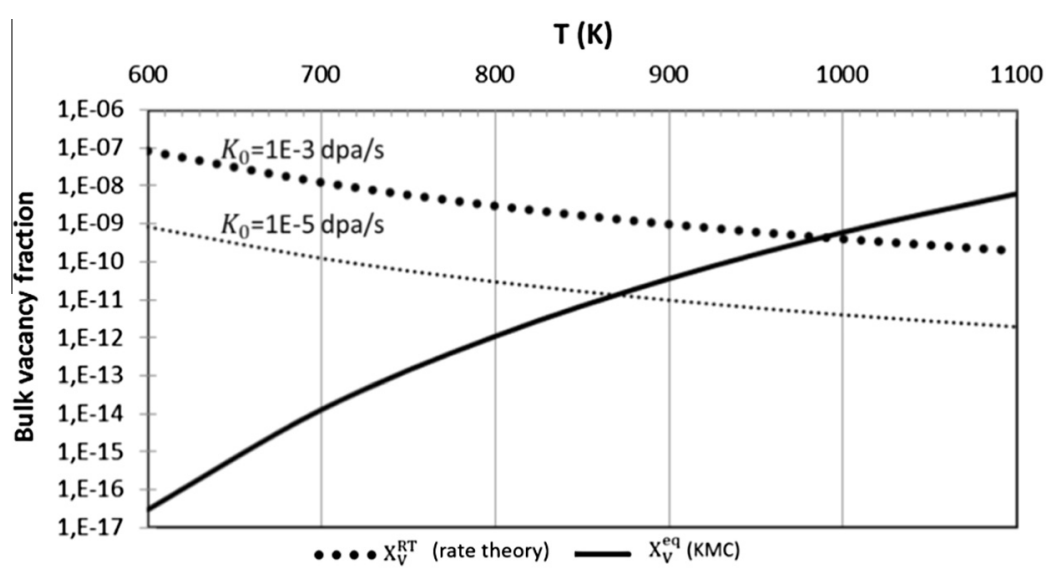

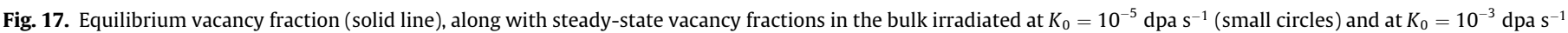
(big circles), as a function of temperature in pure Fe for a sink density $\rho=10^{7} \mathrm{~m}^{-1}$.
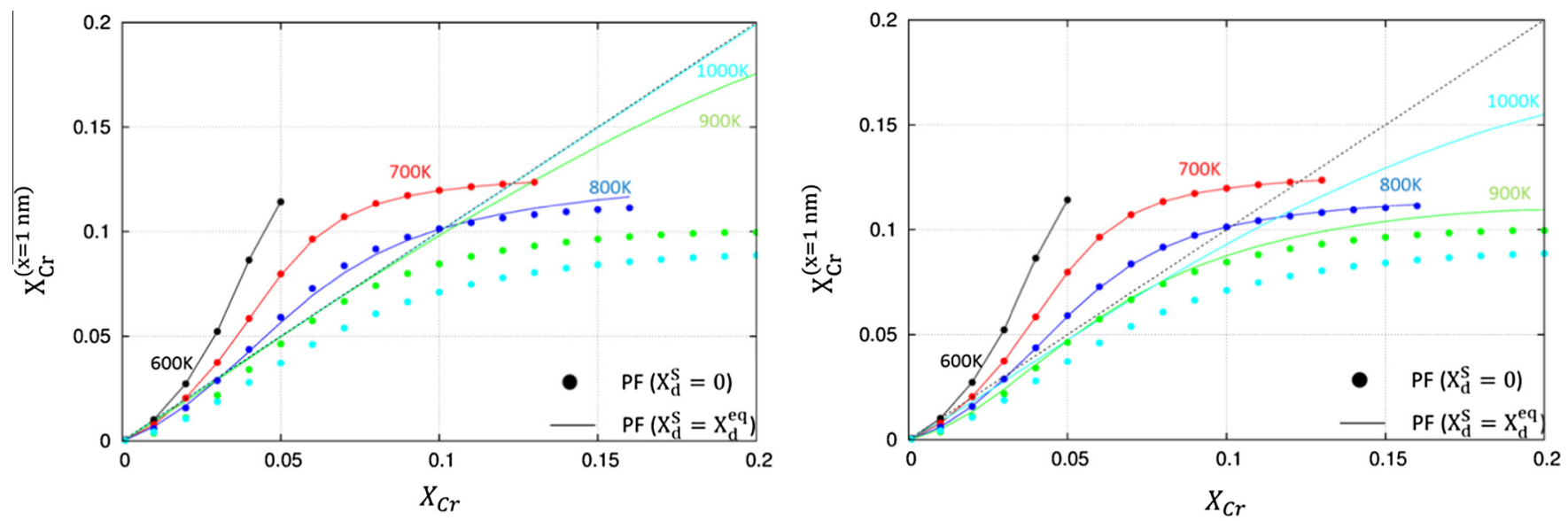

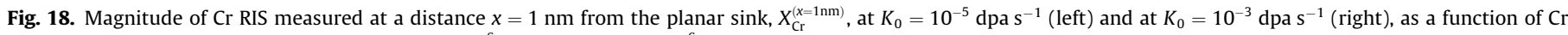
fraction and temperature calculated using PF with $X_{d}^{S}=0$ (symbols) and $X_{d}^{S}=X_{d}^{\text {eq }}$ (lines). The dashed lines represent the limit between Cr depletion and Cr enrichment. 
These simulations therefore clearly evidence the need to take properly into account the composition dependence of both thermodynamic and kinetic parameters in order to obtain quantitative results.

\subsection{Effect of the equilibrium vacancy concentration}

At high temperatures, steady-state PD concentrations no longer exceed the equilibrium concentrations and the PD driving forces tend to zero. For the time being the effect is not taken into account in the AKMC simulations of Section 3 that only include perfect sinks and not the equilibrium mechanisms of PD formation. One must check after the simulation that the steady-state PD concentrations are well above the equilibrium concentrations: if it is not the case (at high temperatures) the RIS predicted by AKMC must be considered as unphysical. The PF model does not suffer such a limitation.

In Section 3, PF calculations were performed in simulation conditions able to mimic AKMC simulations of RIS. In particular, we imposed a zero fraction of PDs at the sink $\left(X_{d}^{S}=0\right)$ in order to reproduce the AKMC simulations. In the more realistic case where $X_{\mathrm{V}}^{S}=X_{\mathrm{V}}^{e q}(r, t)$ in Eq. (20), the thermal fraction of vacancies may play a significant role at high temperatures, according to the value of the PD generation rate, $K_{0}$, and the sink density, $\rho$. Indeed, $X_{\mathrm{V}}^{\text {eq }}$ increases with temperature and can reach a value of the order of $10^{-10}$ as can be seen in Fig. 4. This order of magnitude must be compared to the steady-state fraction of $X_{\mathrm{V}}$ in the bulk of irradiated $\mathrm{Fe}-\mathrm{Cr}$ to determine the conditions of irradiation, sink density and temperatures at which $X_{\mathrm{V}}^{e q}$ may impact significantly the gradients of vacancy fraction profiles at steady state.

The results for $X_{V}^{R T}$ calculated from Eq. (33) are presented in Fig. 17 along with $X_{\mathrm{V}}^{e q}$, as a function of temperature in pure Fe. These results are presented for the sink density used in AKMC simulations (i.e. $\rho=1 / e=10^{7} \mathrm{~m}^{-1}$ ), which corresponds to the smallest order of magnitude which can be considered for the sink density to achieve AKMC calculations in reasonable computational time. In these conditions, thermal vacancies are in the same proportion as the vacancies created by irradiation, at about $880 \mathrm{~K}$ for $K_{0}=10^{-5} \mathrm{dpa} \mathrm{s}^{-1}$ and $1000 \mathrm{~K}$ for $K_{0}=10^{-3} \mathrm{dpa} \mathrm{s}^{-1}$. RIS is therefore expected to be significantly reduced close to or above these temperatures. It can also be mentioned that recombination does not impact $X_{V}^{R T}$ in the whole range of investigated temperatures due to the important sink density which promotes the elimination of PDs.

The results plotted in Fig. 17 are consistent with the results shown in Fig. 18, comparing the magnitude of $\mathrm{Cr}$ segregation at sinks predicted by our PF simulations with $X_{d}^{S}=0$ (symbols) and $X_{d}^{S}=X_{d}^{e q}$ (lines), at $K_{0}=10^{-5} \mathrm{dpa} \mathrm{s}^{-1}$ and $K_{0}=10^{-3} \mathrm{dpa} \mathrm{s}^{-1}$. The segregation trends are barely affected by the value of $X_{d}^{S}$ at temperatures below $800 \mathrm{~K}$ at $K_{0}=10^{-5} \mathrm{dpa} \mathrm{s}^{-1}$, and at temperatures below $900 \mathrm{~K}$ at $K_{0}=10^{-3} \mathrm{dpa} \mathrm{s}^{-1}$. At temperatures above $900 \mathrm{~K}$, RIS is significantly reduced due to the suppression of point defect driving forces at $K_{0}=10^{-3} \mathrm{dpa} \mathrm{s}^{-1}$, and the RIS is almost annihilated at $K_{0}=10^{-5} \mathrm{dpa} \mathrm{s}^{-1}$.

\subsection{Effect of the sink density}

One advantage of PF models against atomic-scale simulations is the possibility to perform calculations with low sink densities, i.e., using large simulations boxes. As an example, we performed RIS simulations at $700 \mathrm{~K}$ for the $\mathrm{Fe}-10 \% \mathrm{Cr}$ alloy at $K_{0}=10^{-5} \mathrm{dpa} \mathrm{s}^{-1}$, for $\rho=10^{7} \mathrm{~m}^{-1}$ and $\rho=2.5 \times 10^{6} \mathrm{~m}^{-1}$ which correspond to a

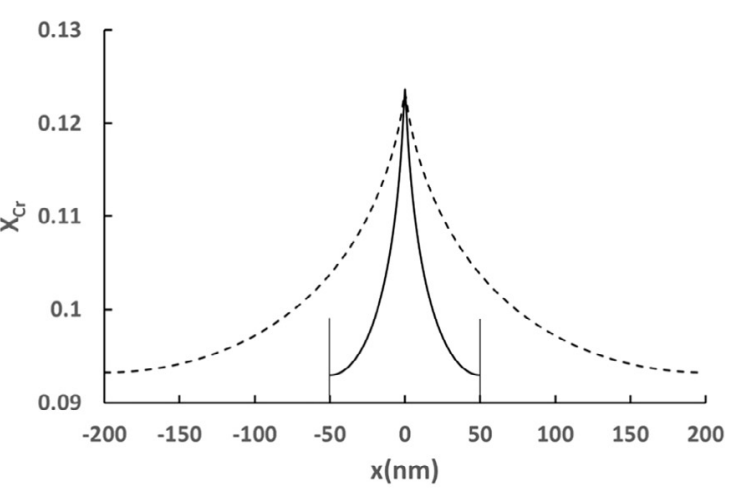

(a)

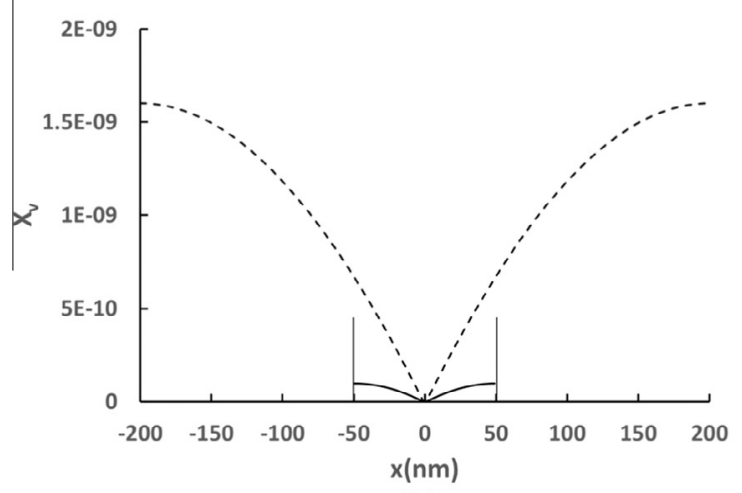

(b)

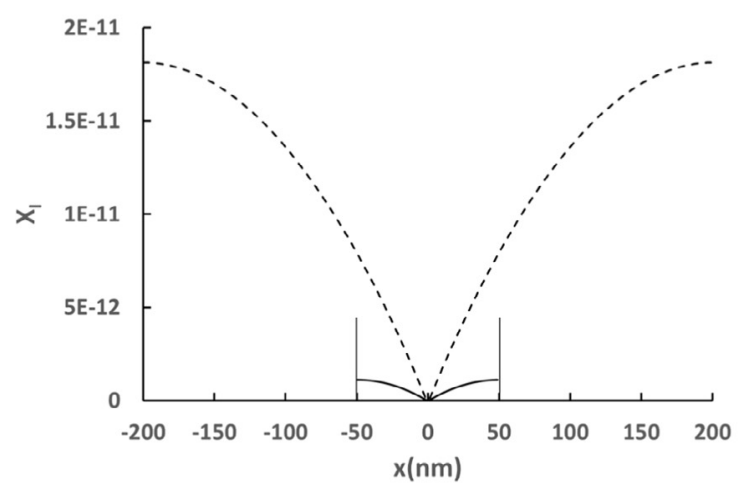

(c)

Fig. 19. $X_{\mathrm{Cr}}$ and $X_{d}$ for a Fe-10\%Cr alloy at $700 \mathrm{~K}$ irradiated at $K_{0}=10^{-5} \mathrm{dpa} \mathrm{s}^{-1}$ in presence of sinks with $\rho=10^{7} \mathrm{~m}^{-1}$ (full lines) and $\rho=2.5 \times 10^{6} \mathrm{~m}^{-1}$ (dashed lines).

box length of $100 \mathrm{~nm}$ and $400 \mathrm{~nm}$ respectively. The RIS and point defect profiles at the steady state are represented in Fig. 19. The PD concentrations far from the sink show a dependence roughly proportional to $K_{d}^{-1}$. What is less expected is the segregation behavior. Indeed, the $\mathrm{Cr}$ profile is wider at low than at high density. This behavior deserves to be studied in more detail in future works.

\section{Conclusions}

The kinetic equations of the Cahn-Hilliard PF method previously derived for the description of interdiffusion phenomena have been extended to describe flux coupling and RIS in alloys. The kinetic equations are written in terms of the Onsager transport coefficients, the equilibrium PD concentrations, the thermodynamic factors and an effective stiffness parameter depending on both the thermodynamic and kinetic parameters of the diffusion 
model. Atomic scale Monte Carlo simulations are used to estimate the parameters of these new phase-field equations with respect to the temperature and the $\mathrm{Cr}$ nominal composition. The resulting RIS predicted by our PF method is in good agreement with direct AKMC simulations of RIS, at all investigated $\mathrm{Cr}$ compositions and temperatures. It has to be mentioned that the CPU time required for the modeling of RIS in Fe-Cr by the PF method is drastically reduced when compared to the AKMC-CPU time: around $10 \mathrm{~min}$ against more than $48 \mathrm{CPU}$-hours for an AKMC simulation.

It is also shown how the PF method can be used to study the effect of each physical parameter separately, in particular the effect of the thermodynamic factor and the equilibrium PD concentration. The stiffness parameter is observed to have a negligible effect on RIS. It is demonstrated that a thermodynamic factor approximated by one leads to an overestimation of RIS. The PF method also takes into account the equilibrium PD concentrations at sinks, leading to the suppression of point defect driving forces and RIS at high temperature. A quantitative prediction of RIS is obtained only if the variations of the transport coefficients and the thermodynamic factor along the RIS profile are taken into account. It is also shown that the typical high temperature regime where RIS disappears is mainly controlled by the equilibrium PD concentration at grain boundaries.

Contrary to the classical phenomenological approach of RIS in concentrated alloys, a PF method combined with a multiscale parameterization based on DFT calculations of PD jump frequencies and AKMC simulations is able to produce quantitative predictions of RIS. The development of this quantitative and efficient PF method allows now to investigate the effect of sinks of various characters and strain on RIS. To deal with precipitation, it could be interesting to use a mixed discrete-continuous description, such as the one introduced in [15].

\section{Acknowledgements}

We thank Enrique Martinez for useful discussions. This research has received partial funding from Eurofusion IReMEV program, it was sponsored by the "NEEDS-Matériaux" project.

\section{References}

[1] G. Was, Fundamentals of Radiation Materials Science, Springer-Verlag, Berlin, 2007.
[2] M. Nastar, F. Soisson, in: Comprensive Nuclear Materials, Elsevier, Oxford, 2012, pp. 471-496.

[3] A.J. Ardell, P. Bellon, Curr. Opin. Solid State Mater. Sci. (2015).

[4] A.R. Allnatt, A.B. Lidiard, Atomic Transport in Solids, Cambridge University Press, 2003.

[5] J.M. Perks, S.M. Murphy, Materials for Nuclear Reactor Core Applications, BNES, London, 1987.

[6] W.G. Wolfer, J. Nucl. Mater. 114 (1983) 292-304.

[7] M.V. Sorokin, A.I. Ryazanov, J. Nucl. Mater. 357 (2006) 82-87.

[8] T. Garnier, V.R. Manga, D.R. Trinkle, M. Nastar, P. Bellon, Phys. Rev. B 88 (2013) 134108.

[9] T. Garnier, M. Nastar, P. Bellon, D.R. Trinkle, Phys. Rev. B 88 (2013) 134201.

[10] T. Garnier, D.R. Trinkle, M. Nastar, P. Bellon, Phys. Rev. B 89 (2014) 144202.

[11] L. Messina, M. Nastar, T. Garnier, C. Domain, P. Olsson, Phys. Rev. B 90 (2014) 104203.

[12] F. Soisson, J. Nucl. Mater. 349 (2006) 235-250.

[13] O. Senninger, F. Soisson, E. Martínez, M. Nastar, C.-C. Fu, Y. Bréchet, Acta Mater. 103 (2016) 1-11.

[14] C. Hin, Y. Bréchet, P. Maugis, F. Soisson, Acta Mater. 56 (2008) 5653-5667.

[15] A. Badillo, P. Bellon, R.S. Averback, Model. Simul. Mater. Sci. Eng. 23 (2015) 35008.

[16] S. Dubey, A. El-Azab, J. Appl. Phys. 114 (2013) 124901.

[17] P. Bellon, in: Compr. Nucl. Mater, Elsevier, Oxford, 2012, pp. 411-432.

[18] J.W. Cahn, J.E. Hilliard, J. Chem. Phys. 28 (1958) 258-267.

[19] M. Nastar, Phys. Rev. B 90 (2014) 144101.

[20] T. Garnier, A. Finel, Y. Le Bouar, M. Nastar, Phys. Rev. B 86 (2012) 54103.

[21] Q. Bronchart, Y.L. Bouar, A. Finel, Phys. Rev. Lett. 100 (2008) 15702.

[22] T. Garnier, M. Nastar, Phys. Rev. B 88 (2013) 134207.

[23] R.E. Stoller, J. Nucl. Mater. 233-237 (Part 2) (1996) 999-1003.

[24] B.D. Wirth, G.R. Odette, D. Maroudas, G.E. Lucas, J. Nucl. Mater. 244 (1997) $185-194$.

[25] K. Arakawa, K. Ono, M. Isshiki, K. Mimura, M. Uchikoshi, H. Mori, Science 318 (2007) 956-959.

[26] A. Prokhodtseva, B. Décamps, A. Ramar, R. Schäublin, Acta Mater. 61 (2013) 6958-6971.

[27] V.A. Ryabov, V.A. Pechenkin, V.L. Molodtsov, D. Terentyev, J. Nucl. Mater. 472 (2016) 43-46.

[28] L. Onsager, Phys. Rev. 37 (1931) 405.

[29] H. Rouchette, L. Thuinet, A. Legris, A. Ambard, C. Domain, Comput. Mater. Sci. 88 (2014) 50-60.

[30] H. Rouchette, L. Thuinet, A. Legris, A. Ambard, C. Domain, Phys. Rev. B 90 (2014) 14104.

[31] H. Rouchette, L. Thuinet, A. Legris, A. Ambard, C. Domain, Nucl. Instrum. Meth. Phys. Res. Sect. B 352 (2015) 31-35.

[32] S. Dubey, A. El-Azab, Comput. Mater. Sci. 106 (2015) 111-122.

[33] M. Nastar, F. Soisson, Phys. Rev. B 86 (2012) 220102.

[34] V. Barbe, M. Nastar, Phys. Rev. B 76 (2007) 54205.

[35] V. Barbe, M. Nastar, Philos. Mag. 87 (2007) 1649-1669.

[36] J.P. Wharry, G.S. Was, Acta Mater. 65 (2014) 42-55.

[37] J.P. Wharry, Z. Jiao, G.S. Was, J. Nucl. Mater. 425 (2012) 117-124.

[38] E. Martínez, O. Senninger, C.-C. Fu, F. Soisson, Phys. Rev. B 86 (2012) 224109. 